\title{
The Full Diagonal Model of a Bose Gas
}

\author{
T.C. Dorlas ${ }^{1}$, J.T. Lewis ${ }^{2}$ and J.V. Pulé ${ }^{3 \star}$ \\ ${ }^{1}$ Department of Mathematics, University College of Swansea, Singleton Park, Swansea SA2 \\ 8PP, Wales, U.K. \\ ${ }^{2}$ School of Theoretical Physics, Dublin Institute of Advanced Studies, 10 Burlington Road, \\ Dublin 4, Ireland \\ ${ }_{3}$ Department of Mathematical Physics, University College, Belfield, Dublin 4, Ireland
}

Received January 29, 1992; in revised form January 20, 1993

\begin{abstract}
This paper is the final one in a series in which we investigate some models of an interacting Bose gas using Varadhan's large deviation version of Laplacian asymptotics; in it we study the equilibrium thermodynamics of the full diagonal model of a Bose gas. We obtain a formula expressing the pressure, in the thermodynamic limit, as the supremum of a functional over the space of positive bounded measures. We analyse this formula for a large class of interaction kernels and show that there is a critical temperature below which there is Bose-Einstein condensation.
\end{abstract}

\section{Introduction}

The Hamiltonian for a system of bosons interacting through a pair potential $\phi\left(x-x^{\prime}\right)$ can be written as

$$
H=T+U
$$

where $T$ is the kinetic energy operator and $U$ is the potential energy operator,

$$
U=\frac{1}{2} \iint \phi\left(x-x^{\prime}\right) \psi^{*}(x) \psi^{*}\left(x^{\prime}\right) \psi(x) \psi\left(x^{\prime}\right) d x d x^{\prime}
$$

where $\psi(x)$ and $\psi^{*}(x)$ satisfy the canonical commutation relations. For particles in a cube $\Lambda$ of volume $V$ in $\mathbb{R}^{d}$ with periodic boundary conditions, the Hamiltonian can be written in terms of momentum space operators using

$$
\begin{gathered}
\psi(x)=\frac{1}{V} \sum_{k} a_{k} e^{i k x} \quad \text { and } \quad v(k)=\int_{\Lambda} \phi(x) e^{-i k x} d x: \\
T=\sum_{k} \varepsilon(k) n_{k}
\end{gathered}
$$

\footnotetext{
* Research Associate, School of Theoretical Physics, Dublin Institute for Advanced Studies
} 
and

$$
U=\frac{1}{2 V} \sum_{q} \sum_{k} \sum_{k^{\prime}} v(q) a_{k+q}^{*} a_{k^{\prime}-q}^{*} a_{k^{\prime}} a_{k},
$$

where $n_{k}=a_{k}^{*} a_{k}$. When the pair-potential $\phi$ is identically zero, the system displays a phase transition, Bose-Einstein condensation, provided the space-dimension $d$ is greater than two: at sufficiently high values of the mean particle density, the ground-state is occupied on a macroscopic scale. Does an analogous phasetransition occur in a system governed by the full Hamiltonian with a non-zero pair-potential? This is one of the major unsolved problems of mathematical physics. The expression (1.4) is complicated and so far the problem has failed to succumb to a direct attack: physical insight must be used to group the terms. It has long been recognized [1] that it is advantageous to write $U$ as a sum $U^{\mathrm{D}}+U^{\mathrm{OD}}$, where $U^{\mathrm{D}}$ can be expressed as a function of the occupation numbers $\left\{n_{k}\right\}$ (the diagonal part and $U^{\mathrm{OD}}$ (the off-diagonal part) cannot be so expressed:

$$
\begin{aligned}
U^{D} & =\frac{v(0)}{2 V}\left(N^{2}-N\right)+\frac{1}{2 V} \sum_{k} \sum_{k^{\prime} \neq k} v\left(k-k^{\prime}\right) n_{k} n_{k^{\prime}}, \\
U^{\mathrm{OD}} & =\frac{1}{2 V} \sum_{k} \sum_{k^{\prime}} \sum_{\substack{q \neq 0 \\
q \neq k-k^{\prime}}} v(q) a_{k+q}^{*} a_{k^{\prime}-q}^{*} a_{k^{\prime}} a_{k} .
\end{aligned}
$$

Here $N=\sum_{k} n_{k}$. Bogoliubov and his school have argued that $U^{D}$ contains terms which deplete the condensate (these terms are claimed to be responsible for a drastic change in the dispersion law of elementary excitations in the long wave-length limit). Huang, Yang and Luttinger [2] have argued that terms in $U$ ? enhance the amount of condensate (there is an instructive "back-of-the-envelope" calculation on p. 156 of Thouless's book [3] which shows that the Huang-YangLuttinger terms $\frac{a}{2 V}\left\{N^{2}-\sum_{k} n_{k}^{2}\right\}$, where $a=v(0)>0$, in $U^{\mathrm{D}}$ give rise to a jumpdiscontinuity in the amount of condensate as the chemical potential increases). Yang and Yang [4] showed that in the case of $d=1$ and $\phi(x)=a \delta(x), a>0$, the grand canonical pressure can be computed explicitly and it exhibits no phase transition (see also [5] and [6]). In this special case, the diagonal part of $U$ reduces to the Huang-Yang-Luttinger terms which at fixed temperature give a non-zero amount of condensate for sufficiently high values of the chemical potential even for $d=1$; it follows that, in this case, the effect of these terms must be cancelled by the off-diagonal terms. It is reasonable to suppose that, in general, the question as to whether condensation occurs in a system governed by the untruncated Hamiltonian $H=T+U$ might be settled by estimating the competing effects of $U^{\mathrm{D}}$ and $U^{\mathrm{OD}}$. The main result of this paper is that the Thouless effect persists in the system governed by the full diagonal Hamiltonian:

$$
H^{\mathrm{FD}}=T+U^{\mathrm{D}} \text {. }
$$

To place this result in context, it is convenient to distinguish four models:

$$
\begin{aligned}
H^{\mathrm{MF}} & =T+\frac{a}{2 V} N^{2}, \quad a>0 ; \\
H^{\mathrm{HYL}} & =H^{\mathrm{MF}}+\frac{a}{2 V}\left\{N^{2}-\sum_{k} n_{k}^{2}\right\} ;
\end{aligned}
$$




$$
\begin{aligned}
H^{\mathrm{PMF}} & =T+\frac{1}{2 V} \sum_{k} \sum_{k^{\prime}} v\left(k-k^{\prime}\right) n_{k} n_{k^{\prime}} ; \\
H^{\mathrm{FD}} & =H^{\mathrm{PMF}}+\frac{a}{2 V}\left\{N^{2}-\sum_{k} n_{k}^{2}\right\} .
\end{aligned}
$$

The first of these models, the meanfield model, has been studied exhaustively; the first rigorous treatment was given by Davies [7]. It was studied in [8] for a more general class of kinetic energy operators and in the present framework in $[9,10$, 11]. The first rigorous treatment of the second model, the Huang-Yang-Luttinger model [2], which we shall refer to as the HYL model, was given in [11, 12] as part of the present programme. The third model, the perturbed meanfield model, was studied in [10] and [13]. The fourth model, the full diagonal model, is the subject of the present paper. In this paper, we express the pressure in the full diagonal model as the infimum of a functional on the space of measures and investigate the properties of the minimizer which are related to Bose-Einstein condensation.

Let $\mathscr{M}_{+}^{b}\left(\mathbb{R}^{d}\right)$ be the space of bounded positive measures on $\mathbb{R}^{d}$; for $\mu \in \mathbb{R}$ let the functional $\mathscr{E}^{\mu}: \mathscr{M}_{+}^{b}\left(\mathbb{R}^{d}\right) \rightarrow \mathbb{R}$ be defined by

$$
\mathscr{E}^{\mu}[m]=f[m]+\frac{1}{2}\langle m, V m\rangle+\frac{1}{2} a\left\{\|m\|^{2}-\sum_{k} m(\{k\})^{2}\right\},
$$

where

$$
\begin{aligned}
f[m] & =\int_{\mathbb{R}^{d}}\|k\|^{2} m(d k)-\beta^{-1}(2 \pi)^{-d} \int_{\mathbb{R}^{d}} s(\rho(k)) d k, \\
s(x) & =(1+x) \ln (1+x)-x \ln x \quad(x \geqq 0),
\end{aligned}
$$

$\rho$ is the Radon-Nikodym derivative of the measure $(2 \pi)^{d} m$ with respect to Lebesgue measure and $\langle m, V m\rangle$ is defined by

$$
\langle m, V m\rangle=\iint_{\mathbb{R}^{d} \times \mathbb{R}^{d}} v(k-k) m(d k) m\left(d k^{\prime}\right) .
$$

In the term $\sum_{k} m(k)^{2}$ the summation is over all $k \in \mathbb{R}^{d}$ : this makes sense because there are at most a countable number of values of $k$ for which $m(k) \neq 0$. We shall prove that $p^{\mathrm{FD}}(\mu)$, the pressure in the full diagonal model for chemical potential $\mu$ in the thermodynamic limit, is given by the variational formula

$$
p^{\mathrm{FD}}(\mu)=-\inf _{\mathscr{M}_{+}^{b}\left(\mathbb{R}^{d}\right)} \mathscr{E}^{\mu}[m] .
$$

It is easy to see how the terms in $\mathscr{E}^{\mu}[m]$ in (1.6) correspond to terms in the Hamiltonian $H^{\mathrm{FD}}$ of the full diagonal model. However, there is a subtle difference: in the expression $\frac{a}{2 V} \sum_{k} n_{k}^{2}$ in the Hamiltonian, the sum is over the fixed eigenmomenta in the cube $\Lambda$, but in the functional $\mathscr{E}^{\mu}[m]$, the corresponding term $\frac{1}{2} a \sum_{k} m(\{k\})^{2}$ involves summation over all the atoms in the measure $m$ and the location of these atoms, if any, in the minimizer is one of the things to be determined in solving the problem. The method used to establish the variational formula is an extension of that used in our earlier papers [10-12]. An upper bound is proved in a straightforward manner using Laplace's method as formulated by Varadhan [14]; the lower bound is more subtle and requires a non-trivial 
extension of the method used in [12] necessitated by the presence of the inhomogeneous term

$$
\frac{a}{2 V} \sum_{k} \sum_{k^{\prime}} v\left(k-k^{\prime}\right) n_{k} n_{k^{\prime}}
$$

This will be descirbed in detail in Sect. 2 .

The variational formula (1.10) established for the first time in this paper enables us to obtain a clear picture of the mechanisms of condensation in the four models whose Hamiltonians were listed above (1.5). In the meanfield model the functional $\mathscr{E}^{\mu}$ in the variational formula $(1.10)$ reduces to

$$
\mathscr{E}_{\mathrm{MF}}^{\mu}(\mu)=f[m]+\frac{1}{2} a\|m\|^{2}-\mu\|m\| .
$$

The entropy term depends only on the absolutely continuous part of the measure, the meanfield term $\frac{1}{2} a\|m\|^{2}$ is independent of the location of any singular part of the measure and the kinetic energy term is smallest when the singular measure is located at $k=0$. It follows that if a minimizer has a singular part it consists solely of an atom at $k=0$. In [10] it was shown that an atom at $k=0$ corresponds to generalized condensation introduced by Girardeau [15] and discussed in [16]. In the absence of the entropy term the kinetic energy term forces the minimizer to be an atom at $k=0$. The presence of the entropy term alters drastically the situation; we showed in [10] that the presence of the entropy term in the functional forces the spreading of a minimizing measure, militating against condensation. However, the Bosonic character of the entropy which makes $s$ strictly increasing leads to an upper bound on the total particle density $\|m\|$ (see [10], Eq. (1.40)). Thus, at low densities, there is no condensation but, when $\|m\|$ exceeds a critical value $\rho_{c}$, the excess particle density $\|m\|-\rho_{c}$ condenses at $k=0$.

In the perturbed meanfield model, the meanfield term $\frac{1}{2} a\|m\|^{2}$ is replaced by $\frac{1}{2}\langle m, V m\rangle$ and the functional $\mathscr{E}^{\mu}$ becomes

$$
\mathscr{E}_{\mathrm{PMF}}^{\mu}[m]=f[m]+\frac{1}{2}\langle m, V m\rangle-\mu\|m\| .
$$

In this case, there is competition between the total energy and the entropy; in general, there is no longer an upper bound on the total particle density $\|m\|$ in the minimizer and the amount of condensate is sensitive to the detailed form of the kernel $v$. In [13] we gave an example in which condensation vanishes when the total density exceeds a critical value.

The HYL model was considered in [11] and [12]. It is possible to gain more insight concerning the mechanism of Bose-Einstein condensation in this model by using the variational formula of this paper; we do this in detail in Sect. 4. In this case, the functional $\mathscr{E}^{\mu}$ reduces to

$$
\mathscr{E}_{\mathrm{HYL}}^{\mu}[m]=f[m]+\frac{1}{2} a\|m\|^{2}+\frac{1}{2} a\left(\|m\|^{2}-\sum_{k} m(\{k\})^{2}\right) .
$$

The term $\frac{1}{2} a\left(\|m\|^{2}-\sum_{k} m(\{k\})^{2}\right)$ corresponds to $\frac{a}{2 V}\left(N^{2}-\sum_{k} n_{k}^{2}\right)$ in the Hamiltonian $H^{\mathrm{HYL}}$. This term is of a purely quantum mechanical character and reflects the Boson statistics. As noted by Thouless [3], this term tends to produce 
condensation since the quadratic form $N^{2}-\sum_{k} n_{k}^{2}$ is minimized when $N=n_{k}$ for some $k$. The presence of the kinetic energy term will tend to favour $k=0$. Thouless showed that a calculation using a rough estimate of the entropy and the term $N^{2}-\sum_{k} n_{k}^{2}$ for the energy leads to a preaiction that the amount of condensate has a jump-discontinuity at a critical value of the chemical potential. The analysis of Sect. 4 shows that there are two regions (see also $[11,12]$ ): one in which the interaction term $\|m\|^{2}-\sum_{k} m(\{k\})^{2}$ dominates and one in which the kinetic energy dominates. It turns out that the parameter describing the strength of the kinetic energy is $\left(p^{\prime \prime}(0)\right)^{-1}$, the reciprocal of the second derivative of the free gas pressure $p$ evaulated at zero chemical potential. For $p^{\prime \prime}(0)<\frac{1}{2 a}$ the kinetic energy term dominates; in the region $p^{\prime \prime}(0)>\frac{1}{2 a}$, the HYL term dominates and it turns out that Thouless's conjecture is correct. In fact, in this region, the dominance of the HYL term results in condensation when $\|m\|$ exceeds a critical value irrespective of the dimension $d$ of momentum space. In the full diagonal model, the functional $\mathscr{E}^{\mu}$ given by (1.6) contains all four terms. It is interesting that, for all the class of kernels $v$ considered in this paper, there is still a region of the parameters in which the HYL term dominates: for a fixed value of the chemical potential, there is a critical temperature below which there is condensation; this result is independent of $d$.

The paper is organized as follows: in Sect. 2, we derive the variational expression for the pressure leaving the parts of the proof which are very technical to Sect. 3; in Sect. 4, we study the variational problem. Besides reexamining in detail the HYL model from the point of view of his paper, we prove that for a class of kernels $v$ and for a fixed chemical potential $\mu$, there is a critical temperature below which the minimizer $m$ always has an atom. We show also that a minimizer cannot have more than one atom. We have not been able to show that if an atom exists in the minimizer it must be located at $k=0$.

\section{A Variational Expression for the Pressure}

In the full diagonal model, as discussed in the introduction, the Hamiltonian is diagonal in the occupation number operators which makes it possible to regard the occupation numbers as random variables rather than as operators. We shall do this using the notation of [13]. The probability space on which we define our random variables is the countable set $\Omega$ of terminating sequences of non-negative integers, an element $\omega$ of $\Omega$ is a sequence $\{\omega(j) \in \mathbb{N}: j=1,2, \ldots\}$ satisfying $\sum_{j \geqq 1} \omega(j)<\infty$. The basic random variables are the occupation numbers $\left\{\sigma_{j}: j=1,2, \ldots\right\}$; they are the evaluation maps $\sigma_{j}: \Omega \rightarrow \mathbb{N}$ defined by $\sigma_{j}(\omega)=$ $\omega(j)$ for each $\omega$ in $\Omega$. The total number of particles in the configuration $\omega$ is defined by

$$
N(\omega)=\sum_{j \geqq 1} \sigma_{j}(\omega) .
$$

Let $\Lambda_{1}, \Lambda_{2}, \ldots$ be a sequence of regions in $\mathbb{R}^{d}$ and denote the volume by $V_{l}$; we assume that $V_{l} \rightarrow \infty$ as $l \rightarrow \infty$. We associate with the region $\Lambda_{l}$ the free-gas Hamiltonian $H_{l}$ given by

$$
H_{l}(\omega)=\sum_{j \geqq 1} \varepsilon\left(k_{l}(j)\right) \sigma_{j}(\omega),
$$


where $\varepsilon: \mathbb{R}^{d} \rightarrow \mathbb{R}$ is a continuous positive map with bounded level sets and $\inf _{k \in \mathbb{R}^{d}} \varepsilon(k)=0$, and $k_{l}(1), k_{l}(2) \ldots$ is a sequence in $\mathbb{R}^{d}$.

The Hamiltonian of the full diagonal model studied in this paper is given by

$$
\begin{aligned}
H_{l}^{\mathrm{FD}}(\omega)= & H_{l}(\omega)+\frac{1}{2 V_{l}} \sum_{j, j^{\prime} \geqq 1} v\left(k_{l}(j), k_{l}\left(j^{\prime}\right)\right) \sigma_{j}(\omega) \sigma_{j^{\prime}}(\omega) \\
& +\frac{a}{2 V_{l}}\left\{N(\omega)^{2}-\sum_{j \geqq 1} \sigma_{j}(\omega)^{2}\right\}
\end{aligned}
$$

where $a=v(0,0)>0$. Since $\Omega$ is a countable set, we may specify a probability measure on $\Omega$ by giving its value at each point of $\Omega$. The free-gas canonical measure is defined for $\mu<0$ by

$$
\mathbb{P}_{l}^{\mu}[\omega]=e^{\beta\left\{\mu N(\omega)-H_{l}(\omega)-V_{l} p_{l}(\mu)\right\}},
$$

provided $\sum_{j \geqq 1} e^{-\beta \varepsilon\left(k_{l}(j)\right)}$ is finite for all $\beta>0$. Here the free-gas pressure $p_{l}(\mu)$, $\mu<0$, is defined by

$$
p_{l}(\mu)=\left(\beta V_{l}\right)^{-1} \ln \sum_{\omega \in \Omega} e^{\beta\{\mu N(\omega)-H(\omega)\}} ;
$$

it is given in terms of $k_{l}(j)$ by

$$
p_{l}(\mu)=\int_{\mathbb{R}^{d}} p(\mu \mid k) v_{l}(d k),
$$

where $v_{l}$ is the measure on $\mathbb{R}^{d}$ defined by

$$
v_{l}(A)=\left(V_{l}\right)^{-1} \#\left\{j: k_{l}(j) \in A\right\}
$$

and $p(\mu \mid k)$ is the partial pressure given by

$$
p(\mu \mid k)=\beta^{-1} \ln \left(1-e^{\beta(\mu-\varepsilon(k))}\right)^{-1} .
$$

The pressure of the full diagonal model is defined by

$$
p_{l}^{\mathrm{FD}}(\mu)=\left(\beta V_{l}\right)^{-1} \ln \sum_{\omega \in \Omega} e^{\beta\left\{\mu N(\omega)-H_{l}^{\mathrm{FD}}(\omega)\right\}},
$$

which we may re-write as

$$
p_{l}^{\mathrm{FD}}(\mu)=p_{l}(\alpha)+\left(\beta V_{l}\right)^{-1} \ln \sum_{\omega \in \Omega} e^{\beta V_{l} g_{l}^{\mu-\alpha}(\omega)} \mathbb{P}_{l}^{\alpha}[\omega]
$$

where $\alpha<0$ and

$$
\begin{aligned}
g_{l}^{\mu-\alpha}(\omega)= & (\mu-\alpha) \frac{N(\omega)}{V_{l}}-\frac{1}{2} \sum_{j, j^{\prime} \geqq 1} v\left(k_{l}(j), k_{l}\left(j^{\prime}\right)\right)\left(\frac{\sigma_{j}(\omega)}{V_{l}}\right)\left(\frac{\sigma_{j^{\prime}}(\omega)}{V_{l}}\right) \\
& -\frac{1}{2} a\left\{\left(\frac{N(\omega)}{V_{l}}\right)^{2}-\sum_{j \geqq 1}\left(\frac{\sigma_{j}(\omega)}{V_{l}}\right)^{2}\right\} .
\end{aligned}
$$

We introduce the occupation measure $\bar{L}_{l}$ by defining for each $\omega$ in $\Omega$,

$$
\bar{L}_{l}[\omega]=\frac{1}{V_{l}} \sum_{j \geqq 1} \sigma_{j}(\omega) \delta_{k_{l}(j)},
$$


where $\delta_{x}$ is the Dirac measure concentrated at $x$ so that for each $\omega$ in $\Omega, \bar{L}_{l}[\omega]$ is a bounded positive measure on $\mathbb{R}^{d}$. We let $\bar{E}=\mathscr{M}_{+}^{b}\left(\mathbb{R}^{d}\right)$ the space of bounded positive measures on $\mathbb{R}^{d}$ equipped with the narrow topology.

As in [12] our first aim is to obtain an upper bound for the interaction term $g_{l}^{\mu-\alpha}(\omega)$ by a functional of the occupation measure $\bar{L}_{l}[\omega]$; to do this we introduce the map $S: \bar{E} \rightarrow \mathbb{R}_{+}$as follows: let

For each $m \in \bar{E}$ let $\Lambda(m)$ be the countable set $\left.\left.\left\{k: k \in \mathbb{R}^{d}, m(\{k\})>0\right\}\right)>0\right\}$ and

$$
S[m]=\sum_{k \in \Lambda(m)} m(\{k\})^{2} .
$$

This definition may appear different from the one used in [12] but in fact one can show that the definition in terms of partitions is equivalent to (2.13).

Define $\bar{G}^{\mu-\alpha}: \bar{E} \rightarrow \mathbb{R}$ by

$$
\bar{G}^{\mu-\alpha}[m]=(\mu-\alpha)\|m\|-\frac{1}{2}\langle m, V m\rangle-\frac{1}{2} a\left\{\|m\|^{2}-S[m]\right\},
$$

where

$$
\langle m, V m\rangle=\int_{\mathbb{R}^{d} \times \mathbb{R}^{d}} v\left(k, k^{\prime}\right) m(d k) m\left(d k^{\prime}\right)
$$

and

$$
\|m\|=\int_{\mathbb{R}^{d}} m(d k)
$$

Then

$$
\bar{G}^{\mu-\alpha}\left[\bar{L}_{l}[\omega]\right] \geqq g_{l}^{\mu-\alpha}(\omega)
$$

with equality for all $\omega$ if and only if all $k_{l}(j)$ are distinct. Thus we have

$$
p_{l}^{\mathrm{FD}}(\mu) \leqq p_{l}(\alpha)+\frac{1}{\beta V_{l}} \ln \sum_{\omega \in \Omega} e^{\beta V_{l} \bar{G}^{\mu-\alpha}\left[\bar{L}_{l}[\omega]\right]} \mathbb{P}_{l}^{\alpha}[d m] .
$$

Let $\overline{\mathbb{K}}_{l}^{\alpha}$ be the probability measure induced on $\bar{E}$ by $\bar{L}_{l}$ :

$$
\overline{\mathbf{K}}_{l}^{\alpha}=\mathbb{P}_{l}^{\alpha} \circ \bar{L}_{l}^{-1} \text {. }
$$

Then we can express the upper bound for $p_{l}^{\mathrm{FD}}$ in terms of $\overline{\mathrm{K}}_{l}^{\alpha}$ :

$$
p_{l}^{\mathrm{FD}}(\mu) \leqq p_{l}(\alpha)+\frac{1}{\beta V_{l}} \ln \int_{\bar{E}} e^{\beta V_{l} \bar{G}^{\mu-\alpha}[m]} \overline{\mathbb{K}}_{l}^{\alpha}[d m] .
$$

We impose conditions on $\left\{k_{l}(j)\right\}$ sufficient to ensure the existence of the limit $p(\alpha)=\lim _{l \rightarrow \infty} p_{l}(\alpha)$; we assume that the following conditions already given in [13] are satisfied: For $\beta>0$, let

$$
m_{l}^{\beta}(d k)=e^{-\beta \varepsilon(k)} v_{l}(d k) .
$$

(T1) There exists a measure $v$ such that for all $\beta>0$,

$$
\int_{\mathbb{R}^{d}} e^{\beta \varepsilon(k)} v(d k)<\infty
$$

and the sequence $\left\{e^{\beta \varepsilon(k)} v_{l}(d k)\right\}$ converges to $e^{\beta \varepsilon(k)} v(d k)$ in the narrow topology. (T2) $v$ is absolutely continuous with respect to Lebesgue measure with a density which is strictly positive almost everywhere. 
If (T1) is satisfied then $p(\mu)=\lim _{l \rightarrow \infty} p_{l}(\mu)$ exists for $\mu<0$ and is given by

$$
p(\mu)=\int_{\mathbb{R}^{d}} p(\mu \mid k) v(d k) .
$$

In the case in which $\varepsilon\left(k_{l}(j)\right), j=1,2, \ldots$ are eigenvalues of the Laplacian with periodic boundary conditions on the cube of side $V_{l}^{1 / d}$ condition (T1) is easily checked (see [13]).

To obtain an upperbound we shall use Laplace's method as formulated by Varadhan ([14] Theorems 3.5 and 3.3); the following version of Varadhan's results is convenient for our purpose:

Varadhan's Theorem [14]. Let $\left\{\mathbb{K}_{l}\right\}$ be a sequence of Radon probability measures on a regular Hausdorff space E satisfying the large deviation principle with rate function $I$ and constants $\left\{a_{l}\right\}$. Suppose $G: E \rightarrow \mathbb{R}$ is upper semicontinuous and satisfies

$$
\lim _{A \rightarrow \infty} \limsup _{l \rightarrow \infty} \frac{1}{a_{l}} \ln \int_{\{x \in E: G(x) \geqq A\}} e^{a_{l} G(x)} \mathbb{K}_{l}[d x]=-\infty,
$$

then,

$$
\limsup _{l \rightarrow \infty} \frac{1}{a_{l}} \ln \int_{E} e^{a_{l} G(x)} \mathbb{K}_{l}[d x] \leqq \sup _{x \in C}\{G(x)-I(x)\} .
$$

Suppose $G: E \rightarrow \mathbb{R}$ is lower semicontinuous and then

$$
\liminf _{l \rightarrow \infty} \frac{1}{a_{l}} \ln \int_{E} e^{a_{l} G(x)} \mathbb{K}_{l}[d x] \geqq \sup _{x \in E}\{G(x)-I(x)\} .
$$

In [10] and [13] we proved a large deviation result for the measures $\left\{\overline{\mathbf{K}}_{l}^{\alpha}\right\}$ (see [13] Theorem 1) which we state here:

Theorem 1. Suppose that (T1) and (T2) hold; then, for each $\alpha<0$ the sequence of probability measures $\left\{\overline{\mathbb{K}}_{l}^{\alpha}\right\}$ satisfies the large deviation principle with constants $\left\{\beta V_{l}\right\}$ and rate function $\bar{I}^{\alpha}: \bar{E} \rightarrow[0, \infty]$ given by

$$
\bar{I}^{\alpha}[m]=f[m]+p(\alpha)-\alpha\|m\|,
$$

where

$$
f[m]=\int_{\mathbb{R}^{d}} \varepsilon(k) m(d k)-\beta^{-1} \int_{\mathbb{R}^{d}} s\left(\frac{d m}{d v}(k)\right) v(d k)
$$

and

$$
s(x)=(1+x) \ln (1+x)-x \ln x \quad(x \geqq 0) .
$$

As in [13] we make the following assumptions on $v$ :

(P) $v: \mathbb{R}^{d} \times \mathbb{R}^{d} \rightarrow \mathbb{R}$ is a bounded, continuous, positive definite function; there exists a continuous, strictly positive, symmetric function $v_{0}: \mathbb{R}^{d} \times \mathbb{R}^{d} \rightarrow \mathbb{R}$ such that for all $m \in \bar{E}$,

$$
\langle m, V m\rangle \geqq\left\langle m, V_{0} m\right\rangle,
$$

where $\left\langle m, V_{0} m\right\rangle=\iint_{\mathbb{R}^{d} \times \mathbb{R}^{d}} v_{0}\left(k, k^{\prime}\right) m(d k) m\left(d k^{\prime}\right)$. 
Note that $(\mathrm{P})$ implies that $a=v(0,0)=\left\langle\delta_{0}, V \delta_{0}\right\rangle \geqq\left\langle\delta_{0}, V_{0} \delta_{0}\right\rangle=v_{0}(0,0)>0$. To apply (2.24), we must check that $m \mapsto \bar{G}^{\mu-\alpha}$ is upper semicontinuous and that it satisfies (2.23); to this end we prove in Sect. 3 the following result

Proposition 1. The functional $S: \bar{E} \mapsto \mathbb{R}_{+}$has the following properties:

1. For each element $m \in \bar{E}$,

$$
S[m] \leqq\|m\|^{2} .
$$

2. The map $m \mapsto S[m]$ is upper semicontinuous on $\bar{E}$.

In Lemma 2.2 of [13] we proved that (2.23) is satisfied by $G^{\mu-\alpha}[m]$ where

$$
G^{\mu-\alpha}[m]=(\mu-\alpha)\|m\|-\frac{1}{2}\langle m, V m\rangle ;
$$

since $S[m] \leqq\|m\|^{2}, \bar{G}^{\mu-\alpha}[m] \leqq G^{\mu-\alpha}[m]$ and so (2.23) is satisfied also by $\bar{G}^{\mu-\alpha}[m]$. Again in [13] we proved that $m \mapsto G^{\mu-\alpha}[m]$ is continuous and therefore by Proposition 1 and the continuity of the map $m \mapsto\|m\|$ on $\bar{E}$ we have that $m \mapsto \bar{G}^{\mu-\alpha}[m]$ is upper semicontinuous. Thus we can apply Varadhan's theorem to get for any closed subset, $C$ of $\bar{E}$ :

$$
\limsup _{l \rightarrow \infty} \frac{1}{V_{l}} \ln \int_{C} e^{V_{l} \bar{G}^{\mu-\alpha}[m]} \overline{\mathbb{K}}_{l}^{\alpha}[d m] \leqq \sup _{m \in C}\left\{\bar{G}^{\mu-\alpha}[m]-\bar{I}^{\alpha}[m]\right\} .
$$

In particular putting $C=\bar{E}$ we obtain an upper bound for the pressure:

$$
\limsup _{l \rightarrow \infty} p_{l}^{F D}(\mu) \leqq p(\alpha)+\sup _{\bar{E}}\left\{\bar{G}^{\mu-\alpha}[m]-\bar{I}^{\alpha}[m]\right\}=-\inf _{\bar{E}} \mathscr{E}^{\mu}[m],
$$

where

$$
\begin{aligned}
\mathscr{E}^{\mu}[m] & =\bar{I}^{\alpha}[m]-\bar{G}^{\mu-\alpha}[m]-p(\alpha) \\
& =f[m]+\frac{1}{2}\langle m, V m\rangle+\frac{1}{2} a\left\{\|m\|^{2}-S[m]\right\}-\mu\|m\| .
\end{aligned}
$$

The proof of the upper bound (2.30) given above was a straightforward generalization of the equivalent proof in [12]; however because of the inhomogeneous interaction term in the model under consideration we cannot employ the results of [11] as in [12] to obtain a lower bound.

For $k_{1}, k_{2}, \ldots, k_{n}$, distinct elements of $\mathbb{R}^{d}$ choose $n$ sequences of positive integers $j(l, i), l=1,2 \ldots, i=1, \ldots n$ such that $\lim _{l \rightarrow \infty} k_{l}(j(l, i))=k_{i}$; because of the assumption (T2) this is always possible. Let $\underline{E}=\mathbb{R}_{+}^{n} \times \bar{E}$ equipped with the product topology and define $\underline{L}_{l}: \Omega \rightarrow \underline{E}$ by

$$
\underline{L}_{l}[\omega]=\left(\frac{\sigma_{j(l, 1)}}{V_{l}}(\omega), \ldots \frac{\sigma_{j(l, n)}}{V_{l}}(\omega), \bar{L}_{l}[\omega]\right) .
$$

If we now define $\underline{G}^{\mu-\alpha}: \underline{E} \rightarrow \mathbb{R}$ by

$$
\underline{G}^{\mu-\alpha}[(x, m)]=(\mu-\alpha)\|m\|-\frac{1}{2}\langle m, V m\rangle-\frac{a}{2}\left(\|m\|^{2}-\sum_{i=1}^{n} x_{i}^{2}\right)
$$


for $x \in \mathbb{R}_{+}^{n}$ and $m \in \bar{E}$, we get a lower bound for $g_{l}^{\mu-\alpha}(\omega)$ :

$$
g_{l}^{\mu-\alpha}(\omega) \geqq \underline{G}^{\mu-\alpha}\left[\underline{L}_{l}[\omega]\right] .
$$

We then proceed as above and let

$$
\underline{\mathbb{K}}_{l}=\mathbb{P}_{l} \circ \underline{L}_{l}^{-1}
$$

to obtain a lower bound for $p_{l}^{\mathrm{FD}}(\mu)$;

$$
p_{l}^{\mathrm{FD}}(\mu) \geqq p_{l}(\alpha)+\left(\beta V_{l}\right)^{-1} \ln \int_{\underline{E}} \exp \left\{\beta V_{l} \underline{G}^{\mu-\alpha}[(x, m)]\right\} \underline{\mathbb{K}}_{l}^{\alpha}[d(x, m)] .
$$

In Sect. 3 we shall prove the following theorem.

Theorem 2. Suppose that (T1) and (T2) are satisfied; then for each $\alpha<0$, the sequence of probability measures $\left\{\underline{\mathbb{K}}_{l}^{\alpha}\right\}$ satisfies the large deviation principle with constants $\left\{\beta V_{l}\right\}$ and rate function $\underline{I}^{\alpha}: \underline{E} \rightarrow[0, \infty]$ given by

$$
\underline{I}^{\alpha}[(x, m)]= \begin{cases}\bar{I}^{\alpha}[m], & \text { if } m\left\{k_{i}\right\} \geqq x_{i}, i=1, \ldots, n \\ \infty & \text { otherwise }\end{cases}
$$

Since $\underline{G}^{\mu-\alpha}[(x, m)]=G^{\mu-\alpha}[m]-\frac{a}{2}\left(\|m\|^{2}-\sum_{i=1}^{n} x_{i}^{2}\right)$ and $m \mapsto G^{\mu-\alpha}[m]$ is continuous, it follows that $(x, m) \mapsto \underline{G}^{\mu-\alpha}[(x, m)]$ is continuous in the product topology on $\underline{E}$. Applying Varadhan's theorem we then get a lower bound for $\liminf _{l \rightarrow \infty} p_{l}^{\mathrm{FD}}(\mu)$ :

$$
\begin{aligned}
\liminf _{l \rightarrow \infty} p_{l}^{\mathrm{FD}}(\mu) \geqq & p(\alpha)+\sup _{\underline{E}}\left\{\underline{G}^{\mu-\alpha}\left[(x, m)-\underline{I}^{\alpha}[(x, m)]\right\}\right. \\
= & -\inf _{\substack{\left.(x, m) \in E \\
m k_{i}\right\} \geqq x_{i}, i=1}}\left\{\int_{\mathbb{R}^{d}}(\varepsilon(k)-\mu) m(d k)+\frac{1}{2}\langle m ; V m\rangle+\frac{1}{2} a\|m\|^{2}\right. \\
& \left.-\frac{1}{2} a \sum_{i=1}^{n} x_{i}^{2}-\beta^{-1} \int_{\mathbb{R}^{d}} s\left(\frac{d m}{d v}(k)\right) v(d k)\right\} \\
= & -\inf _{m \in \bar{E}} \mathscr{E}^{\mu}\left[m ; k_{1}, k_{2}, \ldots k_{n}\right]
\end{aligned}
$$

where

$$
\begin{aligned}
\mathscr{E}^{\mu}\left[m ; k_{1}, k_{2}, \ldots k_{n}\right]= & f[m]+\frac{1}{2}\langle m, V m\rangle \\
& +\frac{1}{2} a\left\{\|m\|^{2}-\sum_{l=1}^{n} m\left(\left\{k_{i}\right\}\right)^{2}\right\}-\mu\|m\| .
\end{aligned}
$$

Since the set $\left\{k_{1}, k_{2}, \ldots, k_{n}\right\}$ is arbitrary we have also

$$
\liminf _{l \rightarrow \infty} p_{l}^{\mathrm{FD}}(\mu) \geqq-\inf _{\left\{k_{1}, \ldots, k_{n}\right\}} \inf _{m \in \bar{E}} \mathscr{E}^{\mu}\left[m ; k_{1}, k_{2}, \ldots k_{n}\right],
$$


where the first infimum is over finite subsets of $\mathbb{R}^{d}$. In Sect. 3 we shall prove that the upper and lower bounds for the pressure are equal, that is:

\section{Proposition 2.}

$$
\inf _{m \in \bar{E}} \mathscr{E}^{\mu}[m]=\inf _{\left\{k_{1}, \ldots, k_{n}\right\}} \inf _{m \in \bar{E}} \mathscr{E}^{\mu}\left[m ; k_{1}, k_{2}, \ldots k_{n}\right]
$$

Combining the inequalities (2.30) and (2.40) we then obtain:

Theorem 3. Suppose that $(T 1)$ and $(T 2)$ hold and that the potential $v$ has the property $(P)$; then the pressure $p^{\mathrm{FD}}(\mu)=\lim _{l \rightarrow \infty} p_{l}^{\mathrm{FD}}(\mu)$ exists for the full diagonal model determined by the Hamiltonian in (2.3), and is given by

$$
p^{\mathrm{FD}}(\mu)=-\inf _{E} \mathscr{E}^{\mu}[m]
$$

where $\mathscr{E}^{\mu}[m]$ is given by (2.31).

\section{Proof of Theorems}

\section{Proof of Proposition 1.}

1. is obvious.

2. For $n \in \mathbb{N}$ let $\left\{\Delta_{j}^{(n)}, j \geqq 1\right\}$ be the countable collection of closed hypercubes of side $n^{-1}$ in $\mathbb{R}^{d}$ of the form $\left\{k: \frac{r_{i}}{n} \leqq k_{i} \leqq \frac{r_{i}+1}{n}, i=1,2, \ldots d\right\}$ with $\left(r_{1}, r_{2}, \ldots r_{d}\right) \in \mathbb{Z}^{d}$. For each $m \in \bar{E}$ let $m_{d}$ denote the discrete part of $m$ and $m_{c}$ the diffuse part, that is

$$
m_{d}=\sum_{k \in \Lambda(m)} m(\{k\}) \delta_{k}
$$

and

$$
m_{c}=m-m_{d} .
$$

The first step in our argument is to prove that

$$
\lim _{n \rightarrow \infty}\left(\sup _{j \geqq 1} m_{c}\left(\Delta_{j}^{(n)}\right)\right)=0 .
$$

Let $C_{N}$ be the closed hypercube of side $2 N$ centred at the origin and suppose that

$$
\limsup _{n \rightarrow \infty}\left(\sup _{j \geqq 1} m_{c}\left(\Delta_{j}^{(n)} \cap C_{N}\right)\right)=\alpha>0
$$

then there is a sequence of hypercubes $\left\{\Delta_{j_{i}}^{\left(n_{2}\right)}\right\}, n_{i} \rightarrow \infty$, such that for $i$ sufficiently large $m_{c}\left(\Delta_{j_{i}}^{\left(n_{2}\right)} \cap C_{N}\right)>\frac{1}{2} \alpha$. For each $i$ choose $k_{i} \in \Delta_{j_{i}}^{\left(n_{i}\right)} \cap C_{N}$; since $\left\{k_{i}\right\} \subset C_{N}$ it has a subsequence which converges to $k_{0}$ say. It then follows that for any open set $O$, containing $k_{0}, m_{c}(O)>\frac{1}{2} \alpha$ and consequently $m_{c}\left(\left\{k_{0}\right\}\right) \geqq \frac{1}{2} \alpha$, which is a contradiction. Therefore

$$
\limsup _{n \rightarrow \infty}\left(\sup _{j \geqq 1} m_{c}\left(\Delta_{j}^{(n)} \cap C_{N}\right)\right)=0 .
$$


Now given $\varepsilon>0$, choose $N$ such that $m_{c}\left(C_{N}^{c}\right)<\varepsilon$; we then have

$$
\sup _{j \geqq 1}\left(m_{c}\left(\Delta_{j}^{(n)}\right)\right) \leqq \sup _{j \geqq 1}\left(m_{c}\left(\Delta_{j}^{n}\right) \cap C_{N}\right)+\varepsilon
$$

and therefore

$$
0 \leqq \liminf _{n \rightarrow \infty}\left(\sup _{j \geqq 1} m_{c}\left(\Delta_{j}^{(n)}\right)\right) \leqq \limsup _{n \rightarrow \infty}\left(\sup _{j \geqq 1}\left(m_{c}\left(\Delta_{j}^{(n)}\right)\right)<\varepsilon .\right.
$$

Since $\varepsilon$ is arbitrary this gives (3.1).

Given $\varepsilon>0$ choose $\Lambda^{\prime} \subset \Lambda(m)$ such that $\#\left(\Lambda^{\prime}\right)<\infty$ and $\sum_{k \in \Lambda(m) \backslash \Lambda^{\prime}} m(\{k\})$ $<\varepsilon\|m\|^{-1} 2^{-(d+2)}$; then choose $n$ such that $n^{-1} d^{\frac{1}{2}}<\min _{\lambda, \lambda^{\prime} \in \Lambda^{\prime}} d\left(\lambda, \lambda^{\prime}\right)$ and $\sup _{j \geqq 1}\left(m_{c}\left(\Delta_{j}^{(n)}\right)\right)<\varepsilon\|m\|^{-1} 2^{-(d+2)}$. With this choice of $n$ we have

$$
\begin{aligned}
\sum_{j \geqq 1} m\left(\Delta_{j}^{(n)}\right)^{2} & \leqq \sum_{j \geqq 1} m_{d}\left(\Delta_{j}^{(n)}\right)^{2}+2^{d+1} \sup _{j \geqq 1}\left(m_{c}\left(\Delta_{j}^{(n)}\right)\right)\|m\| \\
& <\sum_{j \geqq 1} m_{d}\left(\Delta_{j}^{(n)}\right)^{2}+\frac{1}{2} \varepsilon
\end{aligned}
$$

Let $m_{d}^{\prime}=\sum_{k \in \Delta}, m\{k\} \delta_{k}$ and $m_{d}^{\prime \prime}=m_{d}-m_{d}^{\prime}$; then

$$
\begin{aligned}
\sum_{j \geqq 1} m\left(\Delta_{j}^{(n)}\right)^{2} & <\sum_{j \geqq 1} m_{d}^{\prime}\left(\Delta_{j}^{(n)}\right)^{2}+2^{d+1}\|m\|\left\|m_{d}^{\prime \prime}\right\|+\frac{1}{2} \varepsilon \\
& <\sum_{j \geqq 1} m_{d}^{\prime}\left(\Delta_{j}^{(n)}\right)^{2}+\varepsilon=\sum_{k \in \Delta^{\prime}} m(\{k\})^{2}+\varepsilon<S[m]+\varepsilon .
\end{aligned}
$$

Now it is sufficient to prove that $m \mapsto \sum_{j \geqq 1} m\left(\Delta_{j}^{(n)}\right)^{2}$ is upper semicontinuous since then if $\left\{m_{i}\right\}$ is a sequence in $\bar{E}$ converging to $m$ we have

$$
\limsup _{i \rightarrow \infty} S\left(m_{i}\right) \leqq \limsup _{i \rightarrow \infty} \sum_{j \geqq 1} m_{i}\left(\Delta_{j}^{(n)}\right)^{2} \leqq \sum_{j \geqq 1} m\left(\Delta_{j}^{(n)}\right)^{2} \leqq S(m)+\varepsilon
$$

from which the upper semicontinuity of $m \mapsto S(m)$ follows because $\varepsilon$ is arbitrary. We conclude the proof by proving that $m \mapsto \sum_{j \geqq 1} m\left(\Delta_{j}^{(n)}\right)^{2}$ is upper semicontinuous.

First notice that for each $j \geqq 1, m \mapsto m\left(\Delta_{j}^{(n)}\right)$ is upper semicontinuous and therefore $m \mapsto \sum_{j=1}^{J} m\left(\Delta_{j}^{(n)}\right)^{2}$ is upper semicontinuous for any $J<\infty$; we also have that $m \mapsto m\left(\overline{C_{N}^{c}}\right)$ is upper semicontinuous.

Let $\left\{m_{j}\right\}$ be a sequence in $\bar{E}$ converging to $m$. Given $\varepsilon>0$ choose $N$ such that $m\left(\overline{C_{N}^{c}}\right)<\varepsilon$ and let $J$ be such that for $j>J, \Delta_{j}^{(n)} \subset \overline{C_{N}^{c}}$. Then

$$
\sum_{j \geqq 1} m_{i}\left(\Delta_{j}^{(n)}\right)^{2} \leqq \sum_{j=1}^{J} m_{i}\left(\Delta_{j}^{(n)}\right)^{2}+2^{2 d} m_{i}\left(\overline{C_{N}^{c}}\right)^{2},
$$

and thus

$$
\begin{aligned}
\limsup _{i \rightarrow \infty} \sum_{j \geqq 1} m_{i}\left(\Delta_{j}^{(n)}\right)^{2} & \leqq \sum_{j \geqq 1}^{J} m\left(\Delta_{j}^{(n)}\right)^{2}+2^{2 d} \varepsilon^{2} \\
& \leqq \sum_{j \geqq 1} m\left(\Delta_{j}^{(n)}\right)^{2}+2^{2 d} \varepsilon^{2} .
\end{aligned}
$$

The required result follows since $\varepsilon$ is an arbitrary positive number. 
To find a candidate for the rate-function $\underline{I}^{\alpha}$ we employed the following standard trick which was also used to find $\bar{I}^{\alpha}$ (see $\left.[10]\right)$ :

For $y \in \mathbb{R}^{N}$ and $t \in \mathscr{C}^{b}\left(\mathbb{R}^{d}\right)$ let

$$
\underline{C}^{\alpha}[(y, t)]=\lim _{l \rightarrow \infty} \frac{1}{\beta V_{l}} \ln \int_{\underline{E}} e^{\beta V_{l}\langle(x, m),(y, t)\rangle} \underline{\mathbb{K}}_{l}^{\alpha}[d(x, m)] ;
$$

then

$$
\begin{aligned}
& \underline{C}^{\alpha}[(y, t)] \\
& =\lim _{l \rightarrow \infty}\left\{-\frac{1}{\beta V_{l}} \sum_{i=1}^{n} \ln \left(\frac{1-e^{-\beta\left[\varepsilon\left(k_{l}(j(l, i))-\alpha-y_{i}-t\left(k_{l}(j(l, i))\right)\right]\right.}}{1-e^{-\beta\left[\left(\varepsilon\left(k_{l}(j(l, i))\right)-\alpha-t\left(k_{l}(j(l, i))\right)\right]\right.}}\right)+p_{l}[\alpha+t]-p_{l}(\alpha)\right\} \\
& =p[\alpha+t]-p(\alpha),
\end{aligned}
$$

where

$$
p_{l}[\alpha+t]=\int_{\mathbb{R}^{d}} p(\ddot{\alpha}+t(k) \mid k) v_{l}(d k)
$$

and

$$
p[\alpha+t]=\int_{\mathbb{R}^{d}} p(\alpha+t(k) \mid k) v(d k)
$$

provided

$$
\inf _{k \in \mathbb{R}^{d}}\{\varepsilon(k)-\alpha-t(k)\}>0
$$

and

$$
\min _{i=1, \ldots, n}\left\{\varepsilon\left(k_{i}\right)-\alpha-t\left(k_{i}\right)-y_{i}\right\}>0 .
$$

We take $\underline{I}^{\alpha}$ to be the Legendre transform of $\underline{C}^{\alpha}$, that is

$$
\underline{I}^{\alpha}[(x, m)]=\sup _{(y, t)}\left\{\langle(x, m),(y, t)\rangle-\underline{C}^{\alpha}[(y, t)]\right\},
$$

where the supremum is taken over those $(y, t)$ in $\mathbb{R}^{n} \times \mathscr{C}^{b}\left(\mathbb{R}^{d}\right)$ which satisfy (3.10) and (3.11). Then we have

$$
\begin{aligned}
\underline{I}^{\alpha}[(x, m)]= & \sum_{i=1}^{n}\left(\varepsilon\left(k_{i}\right)-\alpha\right) x_{i} \\
& +\sup _{t}\left[\left\langle t, m-\sum_{i=1}^{n} x_{i} \delta_{k_{i}}\right\rangle-p[\alpha+t]+p(\alpha)\right],
\end{aligned}
$$

where the supremum is over $t \in \mathscr{C}^{b}\left(\mathbb{R}^{d}\right)$ satisfying (3.10). If $m\left(\left\{k_{i}\right\}\right)<x_{i}$ for some $i$, we can take $t$ to be large and negătive around $k_{i}$ so that in that case $\underline{I}^{\alpha}[(x, m)]=\infty$; if $m\left(\left\{k_{i}\right\}\right) \geqq x_{i}$ for $i=1, \ldots, n$, using the formula

$$
\bar{I}^{\alpha}[m]=\sup _{t}[\langle t, m\rangle-p[\alpha+t]+p(\alpha)]
$$

we get

$$
\underline{I}^{\alpha}[m]=\sum_{i=1}^{n}\left(\varepsilon\left(k_{i}\right)-\alpha\right) x_{i}+\bar{I}^{\alpha}\left[m-\sum_{i=1}^{n} x_{i} \delta_{k_{i}}\right]=\bar{I}^{\alpha}[m],
$$

by (2.26). 
The proof of Theorem 2 now proceeds in a manner very similar to that of Theorem 3 in [10]. We shall use the notational device of defining $\bar{I}[A]$, where $A$ is a subset of $\bar{E}$, to be the infimum of the set $\{\bar{I}[m]: m \in A\}$ if $A \neq \emptyset$ and $\bar{I}[\emptyset]=\infty$, and similarly for $I$.

Proof of Theorem 2.

1. $\underline{I}^{\alpha}$ is lower semicontinuous because it is the supremum of a family of continuous functions.

2. Let $\bar{K}_{b}=\left\{m \in \bar{E}: \bar{I}^{\alpha}[m] \leqq b\right\}$ and

$$
\underline{K}_{b}=\left\{(x, m) \in \bar{E}, \underline{I}^{\alpha}[(x, m)] \leqq b\right\} .
$$

We want to prove that the level sets of $\underline{I}^{\alpha}, \underline{K}_{b}$ are compact.

$$
\begin{aligned}
\underline{K}_{b} & =\left\{(x, m) \in \underline{E}: m\left(\left\{k_{i}\right\}\right) \geqq x_{i}, i=1, \ldots, n, \bar{I}^{\alpha}[m] \leqq b\right\} \\
& \subset\left\{x: x \in \mathbb{R}^{d}, x_{i} \leqq \sup _{m \in \bar{K}_{b}}\|m\|\right\} \times \bar{K}_{b} .
\end{aligned}
$$

From [10] we know that $\bar{K}_{b}$ is compact and that if $L>0$ is large enough $\bar{K}_{b} \subset B_{L}=\{m \in \bar{E}:\|m\| \leqq L\}$. Thus $\underline{K}_{b}$ is contained in

$$
\left\{x: x \in \mathbb{R}^{d}, 0 \leqq x_{i} \leqq L, i=1, \ldots, n\right\} \times \bar{K}_{b}
$$

which is compact, but since $\underline{I}^{\alpha}$ is lower semicontinuous $\underline{K}_{b}$ is closed and therefore compact.

3. The proof of the large deviation upper bound

$$
\limsup _{l \rightarrow \infty} \frac{1}{\beta V_{l}} \ln K_{l}^{\alpha}[C] \leqq-\underline{I}^{\alpha}[C]
$$

for any closed subset of $E, C$, is exactly as in [10] and we shall omit it.

4. To prove the large deviation lower bound we need the following two results:

Define $\tilde{L}_{l}: \Omega \rightarrow \bar{E}$ by

$$
\tilde{L}_{l}[\omega]=\frac{1}{V_{l}} \sum_{\substack{j \geqq 1 \\ j \neq j \\ i=1, \ldots, i)}} \sigma_{j}(\omega) \delta_{k_{l}(j)},
$$

and let $\tilde{K}_{l}^{\alpha}=\mathbb{P}_{l}^{\alpha} \circ \tilde{L}_{l}^{-1}$. This amounts to removing $n$ elements form the set $\left\{k_{l}(j)\right\}$ and replacing $v_{l}$ by $\tilde{v}_{l}$ where $\tilde{v}_{l}(A)=V_{l}^{-1} \#\left\{j: j \neq j(l, i), i=1, \ldots, n, k_{l}(j) \in A\right\}$. It is clear that $\tilde{v}_{l} \rightarrow v$ in the sense of (T1) and therefore $\tilde{\mathbb{K}}_{l}^{\alpha}$ satisfies the large deviation principle with constants $\left\{\beta V_{l}\right\}$ and rate function $\bar{I}^{\alpha}$.

Define $\hat{L}_{l}: \Omega \rightarrow \mathbb{R}_{+}^{n}$ by

$$
\hat{L}_{l}[\omega]=\left(\frac{\sigma_{j(l, 1)}(\omega)}{V_{l}}, \ldots, \frac{\sigma_{j(l, n)}(\omega)}{V_{l}}\right)
$$

and let

$$
\hat{\mathbb{K}}_{l}^{\alpha}=\mathbb{P}_{l}^{\alpha} \circ \hat{L}_{l}^{-1}
$$

From (2.4) it is easy to see that the random variables $\sigma_{j(l, 1)}(\omega), \ldots, \sigma_{j(l, n)}(\omega)$ are independently distributed according to a geometric distribution with mean 
$\left(\exp \beta\left(\varepsilon\left(k_{l}(j(l, i))\right)-\alpha\right)-1\right)^{-1}$ therefore $\hat{\mathbb{K}}_{l}^{\alpha}$ satisfies the large deviation principle with constants $\left\{\beta V_{l}\right\}$ and rate function

$$
\hat{I}^{\alpha}\left(x_{1}, \ldots, x_{n}\right)=\sum_{i=1}^{n}\left(\varepsilon\left(k_{i}\right)-\alpha\right) x_{i} .
$$

Let $G$ be an open subset of $\underline{E}$. If $\underline{I}^{\alpha}[G]=\infty$ there is nothing to prove; if $\underline{I}^{\alpha}[G]<\infty$ then for each $\varepsilon>0$ there exists $(\bar{x}, \bar{m})$ in $G$ such that

$$
\bar{I}^{\alpha}[\bar{m}]=\underline{I}^{\alpha}(\bar{x}, \bar{m})<\underline{I}^{\alpha}[G]+\varepsilon .
$$

Since $G$ is open there exist $t_{1}, t_{2}, \ldots, t_{r} \in \mathscr{C}^{b}\left(\mathbb{R}^{d}\right)$ and $\delta>0$ such that $G$ contains the neighbourhood $N_{\delta}$ of $(\bar{x}, \bar{m})$ defined by

$$
\begin{gathered}
N_{\delta}=\bigcap_{i=1}^{n}\left\{x \in \mathbb{R}_{+}^{d}:\left|x_{i}-\bar{x}_{i}\right|<\delta\right\} \times \bigcap_{s=1}^{r}\left\{m \in \bar{E}:\left|\left\langle t_{s}, m-\bar{m}\right\rangle\right|<\delta\right\}, \\
\underline{\mathbb{K}}_{l}^{\alpha}\left(N_{\delta}\right)=\mathbb{P}_{l}^{\alpha}\left(\Omega_{1} \cap \Omega_{2}\right),
\end{gathered}
$$

where

$$
\Omega_{1}=\bigcap_{i=1}^{n}\left\{\omega \in \Omega:\left|\frac{\sigma_{j(l, i)}(\omega)}{V_{l}}-\bar{x}_{i}\right|<\delta\right\}
$$

and

$$
\Omega_{2}=\bigcap_{s=1}^{s}\left\{\omega \in \Omega:\left|\frac{1}{V_{l}} \sum_{j \geqq 1} t_{s}\left(k_{l}(j)\right) \sigma_{j}(\omega)-\left\langle t_{s}, \bar{m}\right\rangle\right|<\delta\right\} .
$$

Suppose that for $s=1, \ldots r, \omega \in \Omega$ satisfies

$$
\left|\frac{1}{V_{l}} \sum_{\substack{j \geqq 1 \\ j \neq j(l, i) \\ i=1, \ldots, n}} t_{s}\left(k_{l}(j)\right) \sigma_{j}(\omega)-\left\langle t_{s}, \bar{m}^{\prime}\right\rangle\right|<\frac{\delta}{2},
$$

where $\bar{m}^{\prime}=\bar{m}-\sum_{i=1}^{n} \bar{x}_{i} \delta_{k_{i}}$ and

$$
\left|t_{s}\left(k_{l}(j(l, i))\right) \sigma_{j(l, i)}(\omega)-t_{s}\left(k_{i}\right) \bar{x}_{i}\right|<\frac{\delta}{2 n},
$$

for $i=1, \ldots, n$, then $\omega \in \Omega_{2}$. Now since $t_{s}\left(k_{l}(j(l, i))\right) \rightarrow t_{s}\left(k_{i}\right)$ as $l \rightarrow \infty$ we can find $\delta^{\prime}$ sufficiently small such that if

$$
\left|\frac{\sigma_{j(l, i)}}{V_{l}}-\bar{x}_{i}\right|<\delta^{\prime}, \quad i=1, \ldots n,
$$

then for $l$ large enough $\omega \in \Omega$, and (3.23) is satisfied. Let $\Omega_{1}^{\prime}$ be the set of $\omega \in \Omega$ satisfying (3.24) and $\Omega_{2}^{\prime}$ the set of $\omega \in \Omega$ satisfying (3.22); then $\Omega_{1} \cap \Omega_{2} \supset \Omega_{1}^{\prime} \cap \Omega_{2}^{\prime}$, for $l$ sufficiently large, so that

$$
\begin{aligned}
\mathbb{K}_{l}^{\alpha}\left(N_{\delta}\right) \geqq & \mathbb{P}_{l}^{\alpha}\left(\Omega_{1}^{\prime} \cap \Omega_{2}^{\prime}\right) \\
= & \hat{\mathbb{K}}_{l}^{\alpha}\left(\bigcap_{i=1}^{n}\left\{x \in \mathbb{R}_{+}^{d}:\left|x_{i}-\bar{x}_{i}\right|<\delta^{\prime}\right\}\right) \\
& \times \tilde{\mathbb{K}}_{l}^{\alpha}\left(\bigcap_{s=1}^{r}\left\{m \in \bar{E}:\left|\left\langle t_{s}, m-\bar{m}^{\prime}\right\rangle\right|<\frac{\delta}{2}\right\}\right) .
\end{aligned}
$$


Therefore

$$
\begin{aligned}
\liminf _{l \rightarrow \infty} \frac{1}{\beta V_{l}} \ln \underline{\mathbb{K}}(G) & \geqq \liminf _{l \rightarrow \infty} \frac{1}{\beta V_{l}} \ln \mathbb{K}_{l}^{\alpha}\left(N_{\delta}\right) \\
& \geqq-\hat{I}^{\alpha}\left(x_{1}, \ldots, x_{n}\right)-\bar{I}^{\alpha}\left[\bar{m}^{\prime}\right]=-\bar{I}^{\alpha}[\bar{m}]>-\bar{I}^{\alpha}[G]-\varepsilon ;
\end{aligned}
$$

but $\varepsilon$ was an arbitrary positive number so that the large deviation lower bound holds:

$$
\liminf _{l \rightarrow \infty} \frac{1}{\beta V_{l}} \ln \mathbb{K}_{l}^{\alpha}(G) \geqq-\underline{I}^{\alpha}[G]
$$

Proof of Proposition 2. For any $k_{1}, \ldots, k_{n} \in \mathbb{R}^{d}$, and any $m \in \bar{E}$ we have $S[m] \geqq \sum_{i=1}^{n} m\left(\left\{k_{i}\right\}\right)^{2}$ so that

$$
\mathscr{E}^{\mu}[m] \leqq \mathscr{E}^{\mu}\left[m ; k_{1}, \ldots, k_{n}\right],
$$

and therefore

$$
\inf _{m \in \bar{E}} \mathscr{E}^{\mu}[m] \leqq \inf _{k_{1} \ldots k_{n}} \inf _{m \in \bar{E}} \mathscr{E}^{\mu}\left[m ; k_{1}, \ldots, k_{n}\right] .
$$

Let $\bar{e}=\inf _{m \in \bar{E}} \mathscr{E}^{\mu}[m]$. For any $\varepsilon>0$ there exists $m \in \bar{E}$ such that $\mathscr{E}^{\mu}[m]<\bar{e}+\varepsilon$. Let $\Lambda(m)=\left\{k_{1}, k_{2}, k_{3}, \ldots\right\}$ and put $m_{n}=m_{c}+\sum_{i=1}^{n} m\left(\left\{k_{i}\right\}\right) \delta_{k_{1}}$. Then $m_{n}$ converges to $m$ in the narrow topology and therefore $\frac{1}{2}\left\langle m_{n}, V m_{n}\right\rangle-\frac{1}{2} a\left\|m_{n}\right\|^{2}$ $-\mu\left\|m_{n}\right\|$ converges to $\frac{1}{2}\langle m, V m\rangle-\frac{1}{2} a\|m\|^{2}-\mu\|m\|$. Also

$$
f[m]-f\left[m_{n}\right]=\sum_{i \geqq n+1} \varepsilon\left(k_{i}\right) m\left(\left\{k_{i}\right\}\right)>0
$$

and clearly $S\left(m_{n}\right) \rightarrow S(m)$ as $n \rightarrow \infty$. Thus for $n$ sufficiently large

$$
\mathscr{E}^{\mu}\left[m_{n}\right]<\mathscr{E}^{\mu}[m]+\varepsilon<\bar{e}+2 \varepsilon .
$$

But $\mathscr{E}^{\mu}\left[m_{n}\right]=\mathscr{E}^{\mu}\left[m ; k_{1}, \ldots, k_{n}\right]$ and therefore $\inf _{k_{1} \ldots k_{n}} \inf _{m \in \bar{E}} \mathscr{E}^{\mu}\left[m ; k_{1}, \ldots, k_{n}\right]$ $\leqq \bar{e}+2 \varepsilon$; since $\varepsilon$ is arbitrary we have proved Proposition 2 .

\section{The Minimization Problem and Condensation}

Recall that the pressure for the full diagonal model is given by

$$
p^{\mathrm{FD}}(\mu)=-\inf _{\bar{E}} \mathscr{E}^{\mu}[m],
$$

where

$$
\begin{aligned}
\mathscr{E}^{\mu}[m] & =f[m]+\frac{1}{2}\langle m, V m\rangle+\frac{1}{2} a\left\{\|m\|^{2}-S[m]\right\} \\
& =\bar{I}^{\alpha}[m]-\bar{G}^{\mu-\alpha}[m]-p(\alpha) .
\end{aligned}
$$

Since $\bar{G}^{\mu-\alpha}[m] \leqq G^{\mu-\alpha}[m]$, where $G^{\mu-\alpha}[m]$ is given by (2.29) the proof that the infimum of $\mathscr{E}^{\mu}[m]$ over $\bar{E}$ is attained is exactly as in Lemma 3.1 of [13]; we thus have 
Lemma 4.1. Let $e=\inf \mathscr{E}^{\mu}[m] ;$ then there exists $m^{*} \in \bar{E}$ such that $\mathscr{E}^{\mu}\left[m^{*}\right]=e$.

We can also prove that if $m$ is a minimizer of $\mathscr{E}^{\mu}$ then it must satisfy the following Euler-Lagrange equations:

$$
\begin{gathered}
L^{\mu}(m ; k)=\operatorname{am}(\{k\}) \quad m_{s} \text {-a.e. }, \\
L^{\mu}(m ; k)=\beta^{-1} s^{\prime}(\rho(k)) \quad v \text {-a.e. },
\end{gathered}
$$

where

$$
m(d k)=m_{s}(d k)+\rho(k) v(d k)
$$

is the Lebesgue decomposition of $m$ with respect to $v$ and $L^{\mu}(m ; k)$ is defined by

$$
L^{\mu}(m ; k)=\varepsilon(k)+(V m)(k)+a\|m\|-\mu
$$

with

$$
(V m)(k)=\int_{\mathbb{R}^{d}} v\left(k, k^{\prime}\right) m\left(d k^{\prime}\right) .
$$

The proof of this is as in [10]. However since $\mathscr{E}^{\mu}$ is not convex we cannot prove that Eqs. $(4.1 \mathrm{a}, \mathrm{b})$ are sufficient for $m$ to be a minimizer; in fact we shall see that this is not true in the case of the HYL-model.

Theorem 4. Let $m$ be a minimizer of $\mathscr{E}^{\mu}$ then

(a) $\rho(k)>0$ a.e. with respect to $v$.

(b) $m$ satisfies the Euler-Lagrange equations $(4.1 a, b)$.

In the situation of the perturbed meanfield model studied in [10] and [13], the functional corresponding to $\mathscr{E}^{\mu}$ has a unique minimizer, and if suitable conditions are imposed on $v, v$ and $\varepsilon$ the singular part of the measure is concentrated on the point $k=0$ in momentum space; we were therefore able using Theorem 3.6 of [13] to identify the atom in the minimizer with the generalized Bose-Einstein condensate:

$$
\lim _{\delta \downarrow 0} \lim _{l \rightarrow \infty} \frac{1}{V_{l}} \mathbb{E}\left(\sum_{\left\{j: \varepsilon\left(k_{l}(j)\right) \leqq \delta\right\}} \sigma_{j}\right) .
$$

The situation here is different. As we shall see later when we examine the HYLmodel, $\mathscr{E}^{\mu}$ can have more than one minimizer; this somehow corresponds to the existence of more than one equilibrium state for the system in the thermodynamic limit. Moreover even though we can show that a minimizer can have at most one atom and for low temperatures every minimizer must have an atom we are unable to locate the position of the atom except in simple cases like the HYL model. Therefore we cannot establish mathematically the identification of the weight of the atom in a minimizer with the limit in (4.3) or its equivalent in the case when the atom is not at $k=0$. However since a minimizer represents an equilibrium distribution of the particles according to their momentum we shall still identify the presence of atoms with Bose-Einstein condensation.

It is very instructive to consider the HYL-model analysed in [11] from the point of view of this paper. For the HYL-model, $v\left(k, k^{\prime}\right)=a>0$ for all $k, k^{\prime} \in \mathbb{R}^{d}$ 
and we therefore have

$$
\begin{aligned}
\mathscr{E}^{\mu}[m] & =f[m]+\frac{1}{2} a\left\{2\|m\|^{2}-S[m]\right\} \\
& =\int_{\mathbb{R}^{d}} \varepsilon(k) m(d k)-\mu\|m\|-\frac{1}{\beta} \int_{\mathbb{R}^{d}} s(\rho(k)) v(d k)+\frac{1}{2} a\left\{2\|m\|^{2}-S[m]\right\} .
\end{aligned}
$$

We now assume that $\varepsilon(k)>0$ for all $k \neq 0$ and $\varepsilon(0)=0$. It is clear in that case that if $m$ is a minimizer of $\mathscr{E}^{\mu}[m]$, then $m_{s}$ is concentrated at zero. Let $m$ be a minimizer of $\mathscr{E}^{\mu}$ and let $m_{0}=m(\{0\})$. If $m_{0}=0$ then $m$ must satisfy the Euler-Lagrange equation.

$$
\varepsilon(k)-\mu+2 a\|m\|=\frac{1}{\beta} s^{\prime}(\rho(k)) \quad v \text {-a.e. . }
$$

We shall show that this has a solution if and only if the equation

$$
\frac{\mu-\alpha}{2 a}=p^{\prime}(\alpha)
$$

has a solution $\alpha \leqq 0$.

Suppose (4.4) has a solution then

$$
\left.\rho(k)=e^{\beta(\varepsilon(k)-\mu+2 a\|m\|)}-1\right)^{-1},
$$

and therefore

$$
\|m\|=p^{\prime}(\mu-2 a\|m\|) .
$$

From (4.4) we see that $\varepsilon(k)-\mu+2 a\|m\| \geqq 0 \quad v$-a.e., but $\varepsilon$ is continuous and therefore this must hold for all $k \in \mathbb{R}^{d}$; in particular for $k=0$ we get $2 a\|m\|-\mu \geqq 0$. If we put $\alpha=\mu-2 a\|m\| \leqq 0$ in (4.7) we obtain (4.5).

Conversely if (4.5) has a solution then

$$
m(d k)=\left(e^{\beta(\varepsilon(k)-\alpha)}-1\right)^{-1} v(d k)
$$

satisfies (4.4). Note that in that case

$$
\mathscr{E}^{\mu}[m]=-p_{1}^{\mu}(\alpha),
$$

where

$$
p_{1}^{\mu}(\alpha)=p(\alpha)+(\mu-\alpha)^{2} / 4 a .
$$

Let $\rho_{c}=p^{\prime}(0)$; since $p^{\prime}$ is strictly increasing (4.5) has a unique solution if $\mu \leqq 2 a \rho_{c}$ and no solution if $\mu>2 a \rho_{c}$. Suppose now that $m_{0}>0$ then we have two Euler-Lagrange equations:

$$
\begin{gathered}
-\mu+2 a\|m\|-a m_{0}=0, \\
\varepsilon(k)-\mu+2 a\|m\|=\frac{1}{\beta} s^{\prime}(\rho(k)) \quad v \text {-a.e. } .
\end{gathered}
$$

By letting $\alpha=2 a\|\rho\|-\mu$ it is clear that if $(4.11 \mathrm{a}, \mathrm{b})$ have solution then

$$
\frac{\mu+\alpha}{2 a}=p^{\prime}(\alpha)
$$


has a solution $\alpha$ with $-\mu<\alpha<0$. On the other hand if (4.12) has a solution in $(-\mu, 0)$ then

$$
m(d k)=-\frac{\alpha}{a} \delta_{0}(d k)+\frac{1}{e^{\beta(\varepsilon(k)-\alpha)}-1} v(d k)
$$

is a solution of $(4.11 \mathrm{a}, \mathrm{b})$.

When we examine (4.12) we find the following:

If $\mu>2 a \rho_{c}$ then (4.12) has a unique solution while for $\mu \leqq 2 a \rho_{c}$ the situation is more complicated. If $2 a p^{\prime \prime}(0) \leqq 1$, then for $\mu \leqq 2 a \rho_{c},(4.12)$ does not have a solution in $(-\mu, 0)$. If $2 a p^{\prime \prime}(0)>1$, as in [11] let $\mu_{t}=2 a p^{\prime}\left(\alpha_{t}\right)-\alpha_{t}$, where $\alpha_{t}$ is the unique root of $2 a p^{\prime \prime}(\alpha)=1$, then $\mu_{t}<2 a \rho_{c}$. For $\mu=2 a \rho_{c}$ and $\mu=\mu_{t}$, (4.12) has a unique solution in $(-\mu, 0)$ while for $\mu \in\left(\mu_{t}, 2 a \rho_{c}\right),(4.12)$ has two solutions $\alpha_{1}<\alpha_{2}<0$, say, which must satisfy $p^{\prime \prime}\left(\alpha_{1}\right)<1 / 2 a<p^{\prime \prime}\left(\alpha_{2}\right)$. We shall show that $\alpha_{2}$ does not correspond to a local minimum. To see this fix $x \in \mathbb{R}$ and for $\alpha$ such that $\left(\alpha-\alpha_{2}\right) x>\alpha_{2} / a$ define $m_{\alpha} \in \bar{E}$ by

$$
m_{\alpha}(d k)=\left(-\frac{\alpha_{2}}{a}+\left(\alpha-\alpha_{2}\right) x\right) \delta_{0}(d k)+\frac{1}{e^{\beta(\varepsilon(k)-\alpha)}-1} v(d k),
$$

and let $f(\alpha)=\mathscr{E}^{\mu}\left[m_{\alpha}\right]$. Then $f^{\prime}\left(\alpha_{2}\right)=0$ and

$$
f^{\prime \prime}\left(\alpha_{2}\right)=a x^{2}+4 a p^{\prime \prime}\left(\alpha_{2}\right) x+2 a p^{\prime \prime}\left(\alpha_{2}\right)^{2}+p^{\prime \prime}\left(\alpha_{2}\right) ;
$$

since $p^{\prime \prime}\left(\alpha_{2}\right)>1 / 2 a$ we can choose $x$ such that $f^{\prime \prime}\left(\alpha_{2}\right)<0$.

For $\mu \in\left(\mu_{t}, 2 a \rho_{c}\right)$ let $\alpha^{\prime}=\alpha_{1}$ and for $\mu=2 a \rho_{c}$ and $\mu=\mu_{t}$ let $\alpha^{\prime}$ be the unique solution of (4.12). If

$$
m(d k)=-\frac{\alpha^{\prime}}{a} \delta_{0}(d k)+\frac{1}{e^{\beta\left(\varepsilon(k)-\alpha^{\prime}\right)}-1} v(d k),
$$

then

$$
\mathscr{E}^{\mu}[m]=-p_{2}^{\mu}\left(\alpha^{\prime}\right)
$$

where

$$
p_{2}^{\mu}(\alpha)=(\mu-\alpha)^{2} / 4 a-\alpha^{2} / 2 a+p(\alpha) .
$$

Let $\alpha^{\prime \prime}$ be the unique solution of (4.5). It was shown in [11] that there is a unique value of $\mu, \mu^{*}$ such that

$$
\begin{cases}p_{1}^{\mu}\left(\alpha^{\prime \prime}\right)>p_{2}^{\mu}\left(\alpha^{\prime}\right), & \text { for } \mu_{t} \leqq \mu<\mu^{*} \\ p_{1}^{\mu}\left(\alpha^{\prime \prime}\right)=p_{2}^{\mu}\left(\alpha^{\prime}\right), & \text { for } \mu=\mu^{*}, \\ p_{1}^{\mu}\left(\alpha^{\prime \prime}\right)<p_{2}^{\mu}\left(\alpha^{\prime}\right), & \text { for } \mu_{t}<\mu<2 a \rho_{c} .\end{cases}
$$

We summarize the above results in the following theorem:

Theorem 5. In the HYL-model $\mathscr{E}^{\mu}[m]$ always has a unique minimizer $m^{*}$ except when $2 a p^{\prime \prime}(0)>1$ and $\mu=\mu^{*}$ in which case it has two minimizers $m_{1}^{*}$ and $m_{2}^{*}$.

If $2 a p^{\prime \prime}(0) \leqq 1$ then for $\mu \leqq 2 a \rho_{c}, m^{*}$ is given by (4.8) where $\alpha$ is the unique solution of (4.5) and $\mathscr{E}^{\mu}\left[m^{*}\right]=-p_{1}^{\mu}(\alpha)$, while for $\mu>2 a \rho_{c}, m^{*}$ is given by (4.13), where $\alpha$ is the unique solution of (4.12) and $\mathscr{E}^{\mu}\left[m^{*}\right]=-p_{2}^{\mu}(\alpha)$. 
If $2 a p^{\prime \prime}(0)>1$ then for $\mu<\mu^{*}, m^{*}$ is given by (4.8) where $\alpha$ is the unique solution of (4.5) and $\mathscr{E}^{\mu}\left[m^{*}\right]=-p_{1}^{\mu}(\alpha)$. For $\mu>\mu^{*}, m^{*}$ is given by (4.13) where $\alpha=\alpha^{\prime}$ if $\mu^{*}<\mu \leqq 2 a \rho_{c}$ and $\alpha$ is the unique solution of (4.12) if $\mu>2 a \rho_{c} ; \mathscr{E}^{\mu}[m]=-p_{2}^{\mu}(\alpha)$.

If $\mu=\mu^{*}$ then there are two minimizers: $m_{1}^{*}$ given by (4.8) with $\alpha=\alpha^{\prime \prime}$ and $m_{2}^{*}$ given by (4.13) with $\alpha=\alpha^{\prime} ; \mathscr{E}^{\mu}\left[m_{1}^{*}\right]=-p_{1}^{\mu}\left(\alpha^{\prime \prime}\right)=-p_{2}^{\mu}(\alpha)=\mathscr{E}^{\mu}\left[m_{2}^{*}\right]$.

We shall now treat the general case. We shall assume that $v$ has the properties (A) and (B) below; as we shall see in Proposition 3 and Proposition 4 this results in considerable simplification.

(A) For all $k_{1}, k_{2}$ in $\mathbb{R}^{d}, v$ satisfies the inequality

$$
\left(a+v\left(k_{1}, k_{2}\right)\right)^{2}>v\left(k_{1}, k_{1}\right) v\left(k_{2}, k_{2}\right) .
$$

(B) For all $k_{1}, k_{2}$ in $\mathbb{R}^{d}$, $v$ satisfies the inequality

$$
a+v\left(k_{1}, k_{2}\right)>\frac{1}{2}\left(v\left(k_{1}, k_{1}\right)+v\left(k_{2}, k_{2}\right)\right) \text {. }
$$

Proposition 3. If $v$ satisfies (A) and $m$ is a minimizer of $\mathscr{E}^{\mu}$ then there is at most one $k \in \mathbb{R}^{d}$ such that $m(\{k\})>0$.

Proof. Suppose $m\left(\left\{k_{1}\right\}\right)>0, \quad m\left(\left\{k_{2}\right\}\right)>0$ and $k_{1} \neq k_{2}$. For $x \in \mathbb{R}$ let $\tilde{m}_{\sigma}=m+\sigma x \delta_{k_{1}}+\sigma \delta_{k_{2}}$, where $\sigma \in \mathbb{R}$ is chosen small enough so that $m\left(\left\{k_{1}\right\}\right)+\sigma x>0$ and $m\left(\left\{k_{2}\right\}\right)+\sigma>0$. Let $f(\sigma)=\mathscr{E}^{\mu}\left(\tilde{m}_{\sigma}\right)$; then

$$
f^{\prime}(0)=x\left\{L^{\mu}\left(m ; k_{1}\right)-a m\left(\left\{k_{1}\right\}\right)+\left\{L^{\mu}\left(m ; k_{2}\right)-a m\left(\left\{k_{2}\right\}\right)=0\right.\right.
$$

and

$$
f^{\prime \prime}(0)=x^{2} v\left(k_{1}, k_{1}\right)+2 x\left(v\left(k_{1}, k_{2}\right)+a\right)+v\left(k_{2}, k_{2}\right) .
$$

Now if $m$ is a minimizer we must have $f^{\prime \prime}(0) \geqq 0$ and since $x$ is an arbitrary real number this means that

$$
\left(a+v\left(k_{1}, k_{2}\right)\right)^{2} \leqq v\left(k_{1}, k_{1}\right) v\left(k_{2}, k_{2}\right)
$$

which contradicts (A).

Proposition 4. If $v$ satisfies (B) and $m$ is a minimizer of $\mathscr{E}^{\mu}$ with $m\left(\left\{k_{0}\right\}\right)>0$ for some $k_{0} \in \mathbb{R}^{d}$ then $m$ has no singular continuous part.

Proof. Let $m=m_{0} \delta_{k_{0}}+m^{\prime}, m^{\prime} \in \bar{E}$, and $m^{\prime}\left(\left\{k_{0}\right\}\right)=0$ then

$$
\begin{aligned}
\mathscr{E}^{\mu}(m)= & \left(\varepsilon\left(k_{0}\right)-\mu\right) m_{0}+m_{0}\left(V m^{\prime}\right)\left(k_{0}\right)+a m_{0}\left\|m^{\prime}\right\| \\
& +\frac{1}{2} m_{0}^{2} v\left(k_{0}, k_{0}\right)+\mathscr{E}\left(m^{\prime}\right) .
\end{aligned}
$$

Let $k_{1} \in \mathbb{R}^{d}, m\left(\left\{k_{1}\right\}\right)=0$ and suppose that

$$
\varepsilon\left(k_{1}\right)+\left(V m^{\prime}\right)\left(k_{1}\right)+\frac{1}{2} m_{0} v\left(k_{1}, k_{1}\right)<\varepsilon\left(k_{0}\right)+\left(V m^{\prime}\right)\left(k_{0}\right)+\frac{1}{2} m_{0} v\left(k_{0}, k_{0}\right),
$$

then $\mathscr{E}^{\mu}\left(m_{0} \delta_{k_{1}}+m^{\prime}\right)<\mathscr{E}^{\mu}(m)$ which contradicts the assumption that $m$ is a minimizer; therefore

$$
\varepsilon(k)+\left(V m^{\prime}\right)(k)+\frac{1}{2} m_{0} v(k, k) \geqq \varepsilon\left(k_{0}\right)+\left(V m^{\prime}\right)\left(k_{0}\right)+\frac{1}{2} m_{0} v\left(k_{0}, k_{0}\right)
$$


for all $k \in \mathbb{R}^{d}$ such that $m(\{k\})=0$. Thus if $m\{(k\})=0$,

$$
\begin{aligned}
L^{\mu}(m ; k)= & \varepsilon(k)-\mu+\left(V m^{\prime}\right)(k)+m_{0} v\left(k, k_{0}\right)+a\|m\| \\
\geqq & \varepsilon\left(k_{0}\right)-\mu+\left(V m^{\prime}\right)\left(k_{0}\right)+\frac{1}{2} m_{0} v\left(k_{0}, k_{0}\right) \\
& -\frac{1}{2} m_{0} v(k, k)+m_{0} v\left(k, k_{0}\right)+a\|m\| \\
= & L^{\mu}\left(m ; k_{0}\right)+m_{0} v\left(k_{0}, k_{0}\right)-\frac{1}{2} m_{0}\left(v\left(k_{0}, k_{0}\right)+v(k, k)\right) \\
= & a m_{0}+m_{0} v\left(k, k_{0}\right)-\frac{1}{2} m_{0}\left(v\left(k_{0}, k_{0}\right)+v(k, k)\right),
\end{aligned}
$$

since by (4.1a) $L^{\mu}\left(m ; k_{0}\right)=a m_{0}$. If $v$ satisfies (B) then $L^{\mu}(m ; k)>0$ for every $k \in \mathbb{R}^{d}$ such that $m(\{k\})=0$; this together with (4.1a) implies that the singular part of $m$ must consist of atoms.

Note that if $v\left(k, k^{\prime}\right) \geqq 0$ for all $k, k^{\prime}$ and $v$ satisfies (B) then it satisfies (A). From now on we shall assume that $v\left(k, k^{\prime}\right) \geqq 0$ and that $v$ has the following property:

(P3) For each sequence $\left\{m_{n}\right\}$ which convergences in the narrow topology $\left(V m_{n}\right)(k)$ converges to $(V m)(k)$, where $m=\lim _{n \rightarrow \infty} m_{n}$, uniformly on compact subsets of $\mathbb{R}^{d}$.

For $m \in \bar{E}$ let

$$
L_{0}^{\mu}(m)=\inf _{k \in \mathbb{R}^{d}} L^{\mu}(m ; k) .
$$

Lemma 4.2. Let $\left\{m_{n}\right\}$ be a sequence in $\bar{E}$ such that $m_{n}$ satisfies the Euler-Lagrange equations (4.1) at inverse temperature $\beta_{n}$. Suppose that as $n \rightarrow \infty, \beta_{n} \uparrow \infty$ and $m_{n} \rightarrow m_{0} \in \bar{E}$ in the narrow topology where $m_{0}$ is such that $L_{0}^{\mu}\left(m_{0}\right) \neq 0$. If $\rho_{n}$ is the density of the absolutely continuous part with respect to $v$, of $m_{n}$ then

$$
\lim _{n \rightarrow \infty} \int_{\mathbb{R}^{d}} \rho_{n}(k) v(d k)=0 .
$$

Proof. For each $k \in \mathbb{R}^{d}, m \mapsto L^{\mu}(m ; k)$ is continuous in the narrow topology on $\bar{E}$ and therefore $m \mapsto L_{0}^{\mu}(m)$ is upper semicontinuous; therefore

$$
L_{0}^{\mu}\left(m_{0}\right) \geqq \limsup _{n \rightarrow \infty} L_{0}^{\mu}\left(m_{n}\right) \text {. }
$$

But for each $n, L^{\mu}\left(m_{n}, k\right) \geqq 0 v$-a.e. and therefore since $k \mapsto L^{\mu}\left(m_{n}, k\right)$ is continuous $L^{\mu}\left(m_{n}, k\right) \geqq 0$ for all $k \in \mathbb{R}^{d}$ and so $L_{0}^{\mu}\left(m_{n}\right) \geqq 0$. Choose $\varepsilon>0$ such that $L_{0}^{\mu}\left(m_{0}\right)>\varepsilon>0$. Since $\left\{k: k \in \mathbb{R}^{d}, \varepsilon(k) \leqq 2 \mu\right\}$ is compact there is $n_{0}$ such that for $n>n_{0}$ and $k$ satisfying $\varepsilon(k) \leqq 2 \mu$,

$$
L^{\mu}\left(m_{n} ; k\right) \geqq L^{\mu}\left(m_{0} ; k\right)-\frac{1}{2} \varepsilon>\frac{1}{2} \varepsilon .
$$

But for $k$ satisfying $\varepsilon(k)>2 \mu, L^{\mu}\left(m_{n} ; k\right) \geqq \frac{1}{2} \varepsilon(k)$. Thus using

$$
\int_{\mathbb{R}^{d}} \rho_{n}(k) v(d k)=\int_{\mathbb{R}^{d}} \frac{1}{e^{\beta_{n} L^{\mu}(m ; k)-1}} v(d k)
$$

and (T2) we see that $\lim _{n \rightarrow \infty} \int_{\mathbb{R}^{d}} \rho_{n}(k) v(d k)=0$. 
Lemma 4.3. Let $\left\{m_{n}\right\}$ be a sequence in $\bar{E}$ such that $m_{n}$ satisfies the Euler-Lagrange equations (4.1) at inverse temperature $\beta_{n}$. Let $m_{n}^{a}$ and $m_{n}^{s}$ denote the absolutely continuous and singular parts in the Lebesgue decomposition of $m_{n}$ with respect to $v$. Suppose that as $n \rightarrow \infty, \beta_{n} \uparrow \infty$ and $m_{n}^{a}$ and $m_{n}^{s}$ converge in the narrow topology to $m^{\prime}$ and $m^{\prime \prime}$ respectively then

$$
\liminf _{n \rightarrow \infty} \mathscr{E}^{\mu}\left(m_{n}\right) \geqq \mathscr{E}_{\infty}^{\mu}\left(m_{0}\right)-\int_{\mathbb{R}^{d}} L^{\mu}\left(m_{0} ; k\right) m^{\prime}(d k),
$$

where $m_{0}=m^{\prime}+m^{\prime \prime}$ and for $m \in \bar{E}$,

$$
\mathscr{E}_{\infty}^{\mu}(m)=\int(\varepsilon(k)-\mu) m d k+\frac{1}{2}\langle m, V m\rangle+\frac{1}{2} a\left\{\|m\|^{2}-\frac{1}{2} S(m)\right\} .
$$

Proof.

$$
\begin{aligned}
\frac{1}{\beta_{n}} \int_{\mathbb{R}^{d}} s\left(\rho_{n}(k)\right) v(d k)= & \frac{1}{\beta_{n}} \int_{\mathbb{R}^{d}} \ln \left(1+\rho_{n}(k)\right) v(d k) \\
& +\frac{1}{\beta_{n}} \int_{\mathbb{R}^{d}} \rho_{n}(k) s^{\prime}\left(\rho_{n}(k) v(d k)\right) .
\end{aligned}
$$

By (4.1b)

$$
\frac{1}{\beta_{n}} \int_{\mathbb{R}^{d}} \rho_{n}(k) s^{\prime}\left(\rho_{n}(k)\right) v(d k)=\int_{\mathbb{R}^{d}} L^{\mu}\left(m_{n} ; k\right) m_{n}^{a}(d k)
$$

Thus

$$
\begin{aligned}
\mathscr{E}^{\mu}\left[m_{n}\right]= & \mathscr{E}_{\infty}^{\mu}\left(m_{n}\right)-\int_{\mathbb{R}^{d}} L^{\mu}\left(m_{n}, k\right) m_{n}^{a}(d k)+\frac{1}{\beta_{n}} \int_{\mathbb{R}^{d}} \ln \left(1+\rho_{n}(k)\right) v(d k) \\
= & \int_{\mathbb{R}^{d}}(\varepsilon(k)-\mu) m_{n}^{s}(d k)-\frac{1}{2}\left\langle m_{n}^{a}, V m_{n}^{a}\right\rangle+\frac{1}{2}\left\langle m_{n}^{s}, V m_{n}^{s}\right\rangle \\
& -\frac{1}{2} a\left\|m_{n}^{a}\right\|^{2}+\frac{1}{2} a\left\|m_{n}^{s}\right\|^{2}-\frac{1}{2} a S\left[m_{n}\right]+\frac{1}{\beta_{n}} \int_{\mathbb{R}^{d}} \ln \left(1+\rho_{n}(k)\right) v(d k) .
\end{aligned}
$$

Since $\ln \left(1+\rho_{n}(k)\right) \leqq \rho_{n}(k), \frac{1}{\beta_{n}} \int_{\mathbb{R}^{d}} \ln \left(1+\rho_{n}(k)\right) v(d k) \leqq \frac{1}{\beta_{n}}\left\|m_{n}^{a}\right\|$ and therefore

$$
\lim _{n \rightarrow \infty} \frac{1}{\beta_{n}} \int_{\mathbb{R}^{d}} \ln \left(1+\rho_{n}(k)\right) v(d k)=0 ;
$$

because $m \mapsto \int(\varepsilon(k)-\mu) m(d k)$ is lower semicontinuous, $m \mapsto\langle m, V m\rangle$ is continuous and $m \mapsto S[m]$ is upper semicontinuous we get

$$
\begin{aligned}
\liminf _{n \rightarrow \infty} \mathscr{E}^{\mu}\left[m_{n}\right] \geqq & \int_{\mathbb{R}^{d}}(\varepsilon(k)-\mu) m^{\prime \prime}(d k)-\frac{1}{2}\left\langle m^{\prime}, V m^{\prime}\right\rangle \\
& +\frac{1}{2}\left\langle m^{\prime \prime}, V m^{\prime \prime}\right\rangle-\frac{1}{2} a\left\|m^{\prime}\right\|^{2}+\frac{1}{2} a\left\|m^{\prime \prime}\right\|^{2}-\frac{1}{2} a S\left[m_{0}\right] \\
= & \mathscr{E}_{\infty}^{\mu}\left[m_{0}\right]-\int_{\mathbb{R}^{d}} L^{\mu}\left(m_{0} ; k\right) m^{\prime}(d k) .
\end{aligned}
$$


For the next lemma we require the following notation: For $m \in \bar{E}$ let

$$
C(m)=\left\{k \in \mathbb{R}^{d}: L^{\mu}(m ; k)=L_{0}^{\mu}(m)\right\} .
$$

Lemma 4.4. If $\left\{m_{n}\right\}$ satisfies the conditions of Lemma 4.3 and $m_{0}=m^{\prime}+m^{\prime \prime}$ then supp $m^{\prime} \subset C\left(m_{0}\right)$.

Proof. Since $k \mapsto L^{\mu}\left(m_{0} ; k\right)$ is continuous $C\left(m_{0}\right)$ is closed. Let $k_{0} \in\left(C\left(m_{0}\right)\right)^{c}$ and choose $\delta>0$ such that $B\left(k_{0}, \delta\right) \cap C\left(m_{0}\right)=\emptyset$. Then

$$
\varepsilon \equiv \inf \left\{L^{\mu}\left(m_{0} ; k\right) ; k \in B\left(k_{0}, \frac{1}{2} \delta\right)\right\}>L_{0}^{\mu}\left(m_{0}\right) \geqq 0,
$$

and therefore because of (P3) there is $n_{0}$ such that for $n>n_{0}$,

$$
\inf \left\{L^{\mu}\left(m_{n} ; k\right): k \in B\left(k_{0}, \frac{1}{2} \delta\right)\right\}>\frac{1}{2} \varepsilon .
$$

Consequently by (4.1b) for $n>n_{0}$,

$$
m_{n}^{a}\left(B\left(k_{0}, \frac{1}{2} \delta\right)\right)<\frac{1}{e^{\beta_{n} \varepsilon / 2}-1} v\left(B\left(k_{0}, \frac{1}{2} \delta\right)\right),
$$

and therefore $\lim _{n \rightarrow \infty} m_{n}^{a}\left(B\left(k_{0}, \frac{1}{2} \delta\right)\right)=0$. But then $m^{\prime}\left(B\left(k_{0}, \frac{1}{4} \delta\right)\right)=0$; since $k_{0}$ is arbitrary element of $\left(C\left(m_{0}\right)\right)^{c}$ we have

$$
\left(C\left(m_{0}\right)\right)^{c} \subset \bigcup_{\substack{A \subset \mathbb{R}^{d} \\ \text { open } \\ m^{\prime}(A)=0}} A .
$$

We shall from now on assume that $v$ has the following property:

(P4) There exists a function $u: \mathbb{R}^{d} \rightarrow \mathbb{R}$, strictly positive such that

$$
v\left(k, k^{\prime}\right)=u\left(k-k^{\prime}\right)
$$

for all $k, k^{\prime} \in \mathbb{R}^{d}$.

Note that in this case (A) and (B) are satisfied by $v$. We shall also assume that $\varepsilon(k)>0$ for $k \neq 0$ as we assumed for the HYL-model.

Lemma 4.5. For $\mu>0$ the infimum of $\mathscr{E}_{\infty}^{\mu}$ over $\bar{E}$ is attained at a unique minimizer $m^{*}=\frac{\mu}{a} \delta_{0}$.

Proof. Let $e=\inf _{m \in \bar{E}} \mathscr{E}_{\infty}^{\mu}[m]$; since $\mathscr{E}_{\infty}^{\mu}[0]=0, e \leqq 0$ and therefore there is a sequence $\left\{m_{n}\right\}$ in $\bar{E}$ such that $e \leqq \mathscr{E}_{\infty}^{\mu}\left[m_{n}\right] \leqq 0$ and $\lim _{n \rightarrow \infty} \mathscr{E}_{\infty}^{\mu}\left[m_{n}\right]=e$. Because $\mathscr{E}_{\infty}^{\mu}\left[m_{n}\right] \leqq 0$ for any $\alpha \in \mathbb{R}$ we have:

$$
\begin{aligned}
\int_{\mathbb{R}^{d}}(\varepsilon(k)-\alpha) m_{n}(d k) & \leqq-\frac{1}{2}\left\langle m_{n}, V m_{n}\right\rangle-\frac{1}{2} a\left\{\left\|m_{n}\right\|^{2}-S\left[m_{n}\right)\right\}+(\mu-\alpha)\left\|m_{n}\right\| \\
& \leqq-\frac{1}{2}\left\langle m_{n}, V m_{n}\right\rangle+(\mu-\alpha)\left\|m_{n}\right\| .
\end{aligned}
$$


Let $\alpha<0$, let $\gamma>\mu$ and let $C=\left\{k: k \in \mathbb{R}^{d}, \varepsilon(k) \leqq \gamma\right\}$; then by Lemma 2.1 of [13] it follows that

$$
\int_{\mathbb{R}^{d}}(\varepsilon(k)-\alpha) m_{n}(d k) \leqq \frac{(\mu-\alpha)^{2}}{2 b(C)}+(\mu-\alpha) m_{n}\left(C^{c}\right),
$$

where $b(C)>0$. Thus

$$
(\gamma-\alpha) m_{n}\left(C^{c}\right) \leqq \int_{C^{c}}(\varepsilon(k)-\alpha) m_{n}(d k) \leqq \frac{(\mu-\alpha)^{2}}{2 b(C)}+(\mu-\alpha) m_{n}\left(C^{c}\right) .
$$

Equation (4.28) implies that

$$
(\gamma-\mu) m_{n}\left(C^{c}\right) \leqq \frac{(\mu-\alpha)^{2}}{2 b(C)} ;
$$

therefore the sequence $\left\{m_{n}\left(C^{c}\right)\right\}$ is bounded and thus $\int_{\mathbb{R}^{d}}(\varepsilon(k)-\alpha) m_{n}(d k)$ is bounded. Since $\int_{\mathbb{R}^{d}}(\varepsilon(k)-\alpha) m_{n}(d k) \geqq(-\alpha)\left\|m_{n}\right\|,\left\{\left\|m_{n}\right\|\right\}$ is bounded. Also given $\delta>0$ let $B$ be the compact set $\left\{k: k \in \mathbb{R}^{d}, \varepsilon(k) \leqq \alpha+\delta^{-1}\right\}$, then $m_{n}\left(B^{c}\right) \leqq$ $\delta \int_{\mathbb{R}^{d}}(\varepsilon(k)-\alpha) m_{n}(d k)$ and thus $\left\{m_{n}\right\}$ is uniformly tight. Since $\left\{m_{n}\right\}$ is bounded and uniformly tight, by Prokhorov's Criterion [17] $\left\{m_{n}\right\}$ has a convergent subsequence $\left\{m_{n_{k}}\right\}$. Let $m^{*}=\lim _{k \rightarrow \infty} m_{n_{k}}$; since $\mathscr{E}_{\infty}^{\mu}$ is lower semicontinuous $e \geqq \mathscr{E}_{\infty}^{\mu}\left(m^{*}\right)$ and so $\mathscr{E}_{\infty}^{\mu}$ has a minimizer.

The argument of Proposition 3 applies to $\mathscr{E}_{\infty}^{\mu}$ and so if $m^{*}$ is a minimizer of $\mathscr{E}_{\infty}^{\mu}$ then it has at most one atom; also the argument of Proposition 4 shows that if $m^{*}$ has an atom then $m^{*}$ consists solely of that atom. If $m^{*}$ does not have an atom then $L^{\mu}\left(m^{*} ; k\right)=0, m^{*}$-a.e. and therefore

$$
\int_{\mathbb{R}^{d}}(\varepsilon(k)-\mu) m^{*}(d k)+\left\langle m^{*}, V m^{*}\right\rangle+a\left\|m^{*}\right\|^{2}=0,
$$

so that

$$
\mathscr{E}_{\infty}^{\mu}\left[m^{*}\right]=\frac{1}{2} \int(\varepsilon(k)-\mu) m^{*}(d k) \geqq-\frac{1}{2} \mu\left\|m^{*}\right\|
$$

Now $L^{\mu}\left(m^{*} ; k\right)=0$ implies that $\left\|m^{*}\right\|<\frac{\mu}{\alpha}$ and thus $\mathscr{E}_{\infty}^{\mu}\left[m^{*}\right]>-\frac{\mu^{2}}{2 a}$. But $\mathscr{E}_{\infty}^{\mu}\left[\frac{\mu}{a} \delta_{0}\right]=-\frac{\mu^{2}}{2 a}$ contradicting the assumption that $m^{*}$ is a minimizer of $\mathscr{E}_{\infty}^{\mu}$. Therefore $m^{*}$ consists only of one atom; let $m^{*}=\left\|m^{*}\right\| \delta_{k_{0}}$, then

$$
\mathscr{E}_{\infty}^{\mu}\left[m^{*}\right]=\left(\varepsilon\left(k_{0}\right)-\mu\right)\left\|m^{*}\right\|+\frac{1}{2} a\left\|m^{*}\right\|^{2} .
$$

It is then clear that $m^{*}$ has to be equal to $\frac{\mu}{a} \delta_{0}$.

Lemma 4.6. For each $\beta$ let $m_{\beta} \in \bar{E}$ be a minimizer of $\mathscr{E}^{\mu}$ and let $m_{\beta}=m_{\beta}^{a}+m_{\beta}^{s}$ be the Lebesgue decomposition of $m_{\beta}$ with respect to $v$; then for $\mu>0$, as $\beta \rightarrow \infty$ $m_{\beta}^{a}$ converges to 0 and $m_{\beta}^{s}$ converges to $\frac{\mu}{a} \delta_{0}$ in the narrow topology.

Proof. Let $\beta_{0}>0$ be such that

$$
\int_{\mathbb{R}^{d}} \frac{1}{e^{\beta_{0}(\varepsilon(k)+\mu)}-1} v(d k)<\frac{2 \mu}{a} .
$$


Suppose that $\beta>\beta_{0}, m_{\beta}^{s}=0$ and $\left\|m_{\beta}^{a}\right\| \geqq \frac{2 \mu}{a}$, then $L^{\mu}\left(m_{\beta} ; k\right) \geqq \varepsilon(k)+\mu$ and therefore by (4.1b), $\left\|m_{\beta}^{a}\right\|<\frac{2 \mu}{a}$ giving a contradiction. Thus if $\beta \geqq \beta_{0}$ and $m_{\beta}^{s}=0$ then $\left\|m_{\beta}^{s}\right\|<\frac{2 \mu}{a}$.

If $m_{\beta}^{s} \neq 0$ then by (4.1a), we have $m_{\beta}^{s}$-a.e.:

$$
\varepsilon(k)-\mu+\left(V m_{\beta}\right)(k)+a\left\|m_{\beta}^{a}\right\|=a m_{\beta}(\{k\})-a\left\|m_{\beta}^{s}\right\| \leqq 0 ;
$$

therefore $\left\|m_{\beta}^{s}\right\| \leqq \frac{\mu}{a}$. Thus we have in all cases that if $\beta \geqq \beta_{0}$ then $\left\|m_{\beta}^{s}\right\|<\frac{2 \mu}{a}$. Given $\delta>0$ choose $L$ sufficiently large so that

$$
\int_{\mathbb{R}^{d}} \frac{1}{e^{\frac{1}{2} \beta_{0}(\varepsilon(k)+L)}-1} v(d k)<\delta ;
$$

let $C=\left\{k: \in \mathbb{R}^{d}, \varepsilon(k) \leqq 2 \mu+L\right\}$. Then for $\beta \geqq \beta_{0}, m_{\beta}^{a}\left(C^{c}\right)<\delta$ and so since $C$ is compact $\left\{m_{\beta}^{a}: \beta \geqq \beta_{0}\right\}$ is uniformly tight. The corresponding net of singular measures $\left\{m_{\beta}^{s}: \beta \geqq \beta_{0}\right\}$ is also bounded and uniformly tight since first of all we have $m_{\beta}^{s}$-a.e. that

$$
\varepsilon(k)-\mu+\left(V m_{\beta}\right)(k)+a\left\|m_{\beta}^{a}\right\| \leqq 0,
$$

which means that $\varepsilon(k) \leqq \mu m_{\beta}^{s}$-a.e. or $m_{\beta}^{s}\left\{k: k \in \mathbb{R}^{d}, \varepsilon(k)>\mu\right\}=0$; also (4.30) implies that $\left\langle m_{\beta}^{s}, V m_{\beta}^{s}\right\rangle \leqq \mu\left\|m_{\beta}^{s}\right\|$ so that $\left\|m_{\beta}^{s}\right\| \leqq \frac{\mu}{b}$, where $b=\inf _{\substack{\varepsilon(k) \leqq \mu \\ \varepsilon\left(k^{\prime}\right) \leqq \mu}} v\left(k, k^{\prime}\right)$ $>0$.

Let $\left\{m_{\bar{\beta}}^{-}\right\}$be a subnet of $\left\{m_{\beta}: \beta>\beta_{0}\right\}$, then by Prokhorov's criterion [17] and the Bolzano-Weierstrass theorem there is a countable subnet of $\left\{m_{\bar{\beta}}\right\},\left\{m_{\bar{\beta}_{n}}\right\}$ such that both $\left\{m_{\bar{\beta}_{n}}^{a}\right\}$ and $\left\{m_{\bar{\beta}_{n}}^{s}\right\}$ converge in the narrow topology and $\lim _{n \rightarrow \infty} \bar{\beta}_{n}=\infty$; let $m^{\prime}=\lim _{n \rightarrow \infty} m_{\overline{\beta_{n}}}^{a}$ and $m^{\prime \prime}=\lim _{n \rightarrow \infty} m_{\bar{\beta}_{n}}^{s}$ and let $m_{0}=m^{\prime}+m^{\prime \prime}$. Suppose $L_{0}^{\mu}\left(m_{0}\right)=0$ then by Lemma $4.4, L^{\mu}\left(m_{0} ; k\right)=0, m^{\prime}$-a.e. and so by Lemma 4.3

$$
\liminf _{n \rightarrow \infty} \mathscr{E}^{\mu}\left[m_{n}\right] \geqq \mathscr{E}_{\infty}^{\mu}\left[m_{0}\right]
$$

Now $L^{\mu}\left(\frac{\mu}{a} \delta_{0} ; k\right)=\varepsilon(k)+\frac{\mu}{a} u(k)>0$ for all $k \in \mathbb{R}^{d}$ and therefore $m_{0}$ cannot be equal to $\frac{\mu}{a} \delta_{0}$ so that by Lemma 4.5 ,

$$
\liminf _{n \rightarrow \infty} \mathscr{E}^{\mu}\left[m_{n}\right]>\mathscr{E}_{\infty}^{\mu}\left[\frac{\mu}{a} \delta_{0}\right]=\mathscr{E}^{\mu}\left[\frac{\mu}{a} \delta_{0}\right]
$$

but this means that for $n$ sufficiently large $\mathscr{E}^{\mu}\left[m_{n}\right]>\mathscr{E}^{\mu}\left[\frac{\mu}{a} \delta_{0}\right]$, contradicting the assumption that $\left\{m_{n}\right\}$ are minimizers. Therefore $L_{0}^{\mu}\left(m_{0}\right) \neq 0$ and consequently by Lemma $4.2, m^{\prime}=0$. Since every subnet of $\left\{m_{\beta}^{a}: \beta \geqq \beta_{0}\right\}$ has a subnet which converges to $0, m_{\beta}^{a} \rightarrow 0$. To show that $m_{\beta}^{s} \rightarrow \frac{\mu}{a} \delta_{0}$ let $\left\{m_{\overline{\beta_{n}}}\right\}$ be as above; since we know 
now that $m^{\prime}=0$ by Lemma 4.3 we get

$$
\liminf _{n \rightarrow \infty} \mathscr{E}^{\mu}\left[m_{n}\right] \geqq \mathscr{E}_{\infty}^{\mu}\left[m^{\prime \prime}\right]
$$

If $m^{\prime \prime} \neq \frac{\mu}{a} \delta_{0}$ we get again a contradiction; therefore $m^{\prime \prime}=\frac{\mu}{a} \delta_{0}$ and $m_{\beta}^{s} \rightarrow \frac{\mu}{a} \delta_{0}$.

Theorem 6. Suppose that $v$ has the properties $(P 3)$ and $(P 4)$ and that $\varepsilon(k)>0$ for $k \neq 0$. Then there is a value of the inverse temperature $\beta_{0}$ such that if $\beta>\beta_{0}$, every minimizer of $\mathscr{E}^{\mu}$ is the sum of a measure which is absolutely continuous with respect to $v$ and a single atom; moreover for each $\varepsilon>0$ there is $a \beta_{1}>\beta_{0}$ such that if $\beta>\beta_{1}$ and $m=m_{0} \delta_{k_{0}}+m_{a}$ is a minimizer of $\mathscr{E}^{\mu}$, where $m_{0}>0, k_{0} \in \mathbb{R}^{d}$ and $m_{a} \in \bar{E}$ is absolutely continuous with respect to $v$, then $\left|m_{0}-\frac{\mu}{a}\right|<\varepsilon,\left\|k_{0}\right\|<\varepsilon$ and $\left\|m_{a}\right\|<\varepsilon$.

Proof. We know from Proposition 3 that a minimizer can have at most one atom and from Proposition 4 that if it has an atom then its continuous part is absolutely continuous with respect to $v$. Suppose there is no value of the inverse temperature $\beta_{0}$ such that for $\beta>\beta_{0}$ every minimizer of $\mathscr{E}^{\mu}$ has an atom; then there is a sequence of minimizers $\left\{m_{n}\right\}$ at inverse temperatures $\left\{\beta_{n}\right\}$ such that $\lim _{n \rightarrow \infty} \beta_{n}=\infty$ and each $m_{n}$ is continuous. Let $m_{n}^{s}$ be the singular part of $m_{n}$; by Eq. (4.1a), $L^{\mu}\left(m_{n}^{s} ; k\right)=0, m_{n}^{s}$-a.e. and therefore

$$
-\mu\left|m_{n}^{s}\right|+\left\langle m_{n}^{s}, V m_{n}\right\rangle+a\left\|m_{n}\right\|\left\|m_{n}^{s}\right\| \leqq 0 .
$$

Now by Lemma 4.6 both $m_{n}^{s}$ and $m_{n}$ converge to $\frac{\mu}{a} \delta_{0}$ as $n \rightarrow \infty$; letting $n \rightarrow \infty$ in (4.31) we get a contradiction: $\frac{\mu^{2}}{a} \leqq 0$.

The rest of the theorem is an immediate consequence of Lemma 4.6.

We note that we have not excluded the possibility that singular part of a minimizer $m^{*}$ of $\mathscr{E}^{\mu}$ can be continuous; we have only excluded the coexistence of a singular continuous part with an atomic part. However if we make the assumptions of rotation invariance (R1), (R2) (R3) in [13] and assume also that the smoothness condition on $v$ in Lemma 3.3 of [13] and the condition on $v$ in Theorem 4 of [13] then in the case when $m^{*}(\{k\})=0$ for all $k \in \mathbb{R}^{d}$, the argument of Theorem 4 in that paper is valid and so the measure $m^{*}$ is absolutely continuous with respect to $v$.

We now proceed to check that a kernel $v$ which is the Fourier transform of a positive translation invariant pair-interaction has the properties necessary for Theorem 6 to hold apart from the positivity condition in (P4) which has to be assumed separately; in particular it satisfies (P3).

Let $\phi: \mathbb{R}^{d} \rightarrow \mathbb{R}$ be strictly positive and integrable; for $k \in \mathbb{R}^{d}$ let $u(k)=$ $\frac{1}{(2 \pi)^{d / 2}} \int_{\mathbb{R}^{d}} e^{i k r} \phi(r) d r$, where $d r$ is Lebesgue measure on $\mathbb{R}^{d}$. Let $v\left(k, k^{\prime}\right)=u\left(k-k^{\prime}\right)$, then $v$ is a bounded, continuous, strictly positive definite function. In addition we have:

Proposition 5. The kernel $v$ defined above has the property $(P 3)$.

Proof. Let $\left\{m_{n}\right\}$ be a sequence in $\bar{E}$ and suppose that $m_{n}$ converges in the narrow topology to $m \in \bar{E}$. Then $\lim _{n \rightarrow \infty}\left\|m_{n}\right\|=\|m\|$ and for any $b>0$ satisfying 
$m\left\{k \in \mathbb{R}^{d}:\|k\|=b\right\}=0$ we have for $\left(i_{1}, i_{2}, \ldots, i_{d}\right) \in \mathbb{N}^{d}$ that

$$
\lim _{n \rightarrow \infty} \int_{\|k\| \leqq b}\left(k_{1}^{i_{1}} k_{2}^{i_{2}} \ldots k_{d}^{i_{d}}\right) m_{n}(d k)=\int_{\|k\| \leqq b}\left(k_{1}^{i_{1}} k_{2}^{i_{2}} \ldots k_{d}^{i_{d}}\right) m(d k) .
$$

Let $R>0$ and $\varepsilon>0$. Choose $R_{0}>0$ such that $\mid\left(u(k) \mid<\varepsilon\right.$ if $\|k\|>R_{0}$; this is possible by the Riemann-Lebesgue lemma. Choose $b>R_{0}+R$ such that $m\left\{k: k \in \mathbb{R}^{d},\|k\|=b\right\}=0$; choose $r_{1}>0$ such that $\int_{\|\boldsymbol{r}\|>r_{1}} \phi(r) d r<\varepsilon$. Finally choose $N \in \mathbb{N}$ such that $\sum_{s=N}^{\infty} \frac{\left(b r_{1}\right)^{s}}{s !}<\varepsilon$. For $\|k\| \leqq R$,

$$
\begin{aligned}
\left|\left(V m_{n}\right)(k)-(V m)(k)\right| \leqq & \left|\int_{\left\|k^{\prime}\right\| \leqq b} u\left(k-k^{\prime}\right) m_{n}(d k)-\int_{\left\|k^{\prime}\right\| \leqq b} u\left(k-k^{\prime}\right) m\left(d k^{\prime}\right)\right| \\
& +\int_{\left\|k^{\prime}\right\|>b}\left|u\left(k-k^{\prime}\right)\right| m_{n}(d k)+\int_{\left\|k^{\prime}\right\|>b}\left|u\left(k-k^{\prime}\right)\right| m\left(d k^{\prime}\right) .
\end{aligned}
$$

Now if $\|k\| \leqq R$ and $\left\|k^{\prime}\right\|>b,\left\|k-k^{\prime}\right\|>b-R>R_{0}$ and therefore $\left|u\left(k-k^{\prime}\right)\right|$ $<\varepsilon$; thus

$$
\int_{\left\|k^{\prime}\right\|>b}\left|u\left(k-k^{\prime}\right)\right| m_{n}\left(d k^{\prime}\right)<\varepsilon\left\|m_{n}\right\|
$$

and

$$
\begin{aligned}
& \int_{\left\|k^{\prime}\right\|>b}\left|u\left(k-k^{\prime}\right)\right| m\left(d k^{\prime}\right)<\varepsilon\|m\| . \\
& \int_{\left\|k^{\prime}\right\| \leqq b} u\left(k-k^{\prime}\right) m\left(d k^{\prime}\right)= \int_{\mathbb{R}^{d}} e^{i k r}\left(\int_{\left\|k^{\prime}\right\|<b} e^{-i k^{\prime} r} m\left(d k^{\prime}\right)\right) \phi(r) d r \\
&= \int_{\|\boldsymbol{r}\| \leqq r_{1}} e^{i k r}\left(\int_{\left\|k^{\prime}\right\| \leqq b} e^{-i k^{\prime} r} m\left(d k^{\prime}\right)\right) \phi(r) d r \\
&+\int_{\|r\|>r_{1}} e^{i k r}\left(\int_{\left\|k^{\prime}\right\| \leqq b} e^{-i k^{\prime} r} m\left(d k^{\prime}\right)\right) \phi(r) d r .
\end{aligned}
$$

The modulus of the second term is clearly bounded by $\varepsilon\|m\|$; we split up the first term again:

$$
\begin{aligned}
\int_{\|r\| \leqq r_{1}} e^{i k r}\left(\int_{\left\|k^{\prime}\right\| \leqq b} e^{-i k^{\prime} r} m\left(d k^{\prime}\right)\right) \phi(r) d r \\
=\int_{\|r\| \leqq r_{1}} e^{i k r}\left(\int_{\left\|k^{\prime}\right\| \leqq b}\left(\sum_{s=0}^{N-1} \frac{\left(-i k^{\prime} r\right)^{s}}{s !}\right) m\left(d k^{\prime}\right)\right) \phi(r) d r \\
\quad+\int_{\|r\| \leqq r_{1}} e^{i k r}\left(\int_{\left\|k^{\prime}\right\| \leqq b}\left(\sum_{s=N}^{\infty} \frac{\left(-i k^{\prime} r\right)^{s}}{s !}\right) m\left(d k^{\prime}\right)\right) \phi(r) d r .
\end{aligned}
$$

The modulus of the second term is bounded by

$$
\int_{\mathbb{R}^{d}} \phi(r) d r\|m\|\left(\sum_{s=N}^{\infty} \frac{\left(b r_{1}\right)^{s}}{s !}\right)<\varepsilon\|m\|\|\phi\|_{1} .
$$


Repeating these bounds with $m$ replaced by $m_{n}$ and combining them we get for $\|k\| \leqq R$ :

$$
\begin{aligned}
& \left|\left(V m_{n}\right)(k)-(V m)(k)\right| \\
& \qquad \sum_{s=0}^{N} \frac{1}{s !}\left|\int_{\|r\|<r_{1}} e^{i k r} \phi(r)\left(\int_{\left\|k^{\prime}\right\| \leqq b}\left(k^{\prime} \cdot r\right)^{s} m_{n}\left(d k^{\prime}\right)-\int_{\left\|k^{\prime}\right\| \leqq b}\left(k^{\prime} \cdot r\right)^{s} m\left(d k^{\prime}\right)\right) d r\right| \\
& \quad+\varepsilon\left(2+\|\phi\|_{1}\right)\left(\|m\|+\left\|m_{n}\right\|\right) \\
& \leqq \sum_{s=0}^{N} \frac{1}{s !}\|\phi\|_{1} r_{1}^{s} \sum_{i_{1}+i_{2}+\ldots+i_{d}=s} \mid \int_{\left\|k^{\prime}\right\| \leqq b}\left(k_{1}^{\prime i_{1}} k_{2}^{\prime i_{2}} \ldots k_{d}^{\prime i_{d}}\right) m_{n}\left(d k^{\prime}\right) \\
& \quad-\int_{\left\|k^{\prime}\right\| \leqq b}\left(k_{1}^{\prime i_{1}} k_{2}^{\prime i_{2}} \ldots k_{d}^{i_{d}}\right) m\left(d k^{\prime}\right) \mid+\varepsilon\left(2+\|\phi\|_{1}\right)\left(\|m\|+\left\|m_{n}\right\|\right) .
\end{aligned}
$$

Thus using (4.32) we see that $\left|\left(V m_{n}\right)(k)-(V m)(k)\right|$ can be made arbitrarily small for $n>n_{0}$ where $n_{0}$ is sufficiently large but can be chosen independent of $k$ for $\|k\| \leqq R$.

\section{References}

1. Lee, T.D., Yang, C.N.: The many-body problem in quantum mechanics and quantum statistical mechanics. Phys. Rev. 105, 1119-1120 (1957)

2. Huang, K., Yang, C.N., Luttinger, J.M.: Imperfect Bose gas with hard-sphere interactions. Phys. Rev. 105, 776-784 (1957)

3. Thouless, D.J.: The quantum mechanics of many-body systems. New York: Academic Press 1961

4. Yang, C.N., Yang, C.P.: Thermodynamics of a one-dimensional system of bosons with repulsive delta-function interaction. J. Math. Phys. 10, 1115-1122 (1969)

5. Dorlas, T.C., Lewis, J.T., Pulé, J.V.: The Yang-Yang thermodynamic formalism and large deviations. Commun. Math. Phys. 124, 365-402 (1984).

6. Dorlas, T.C.: Orthogonality and completeness of the Bethe ansatz eigenstates of the nonlinear Schrödinger model. To appear in Commun. Math. Phys. (1993)

7. Davies, E.B.: The thermodynamic limit for an imperfect boson gas. Commun. Math. Phys. 28, 69-86 (1972)

8. van den Berg, M., Lewis, J.T., de Smedt, Ph: Condensation in the imperfect boson gas. J. Stat. Phys. 37, 697-707 (1984)

9. Lewis, J.T., Pulé, J.V., Zagrebnov, V.A.: The large deviation principle for the Kac distribution. Helv. Phys. Acta. 61, 1063-1078 (1988)

10. van den Berg, M., Dorlas, T.C., Lewis, J.T., Pulé, J.V.: A perturbed meanfield model of an interacting boson gas and the large deviation principle. Commun. Math. Phys. 127, 41-69 (1990)

11. van den Berg, M., Lewis, J.T., Pulé, J.V.: The large deviation principle and some models of an interacting boson gas. Commun. Math. Phys. 118, 61-85 (1988)

12. van den Berg, M., Dorlas, T.C., Lewis, J.T., Pulé, J.V.: The pressure in the Huang-YangLuttinger model of an interacting boson gas. Commun. Math. Phys. 128, 231-245 (1990)

13. Dorlas, T.C., Lewis, J.T., Pulé, J.V.: Condensation in some perturbed meanfield models of a Bose gas. Helv. Phys. Acta 64, 1200-1224 (1991)

14. Varadhan, S.R.S.: Asymptotic probabilities and differential equations. Commun. Pure Appl. Math. 19, 261-286 (1966) 
15. Girardeau, M.: Relationship between systems of inpenetrable bosons and fermions in one dimension. J. Math. Phys. 1, 516-523 (1960)

16. van den Berg, M., Lewis, J.T., Pulé, J.V.: A general theory of Bose-Einstein condensation. Helv. Phys. Acta. 59, 1271-1288 (1986)

17. Bourbaki, N., Éléments de mathématiques, chap IX: Intégration. Paris: Hermann 1969

Communicated by J. Fröhlich 
